\title{
Causality between Inflation and Inflation Uncertainty in South Africa: Evidence from a Markov-Switching Vectorautoregressive model
}

Adnen Ben Nasr ${ }^{\#}$, Mehmet Balcilar $\$$, Ahdi N. Ajmi ${ }^{\star}$, Goodness C. Aye ${ }^{*}$, Rangan Gupta* and

Reneé van Eyden*

\begin{abstract}
This study investigates the asymmetric and time-varying causality between inflation and inflation uncertainty in South Africa within a conditional Gaussian Markov switching vector autoregressive (MS-VAR) model framework. The MS-VAR model is capable of determining both the sign and direction of causality. We account for the nonlinear, long memory and seasonal features of inflation series simultaneously by measuring inflation uncertainty as the conditional variance of inflation generated by recursive estimation of a Seasonal Fractionally Integrated Smooth Transition Autoregressive Asymmetric Power GARCH (SEA-FISTAR-APGARCH) model using monthly data for the period 1921:01 to 2012:12. The recursive, rather than a full-sample, estimation allows us to obtain a time-varying measure of uncertainty and better mimics the real-time scenario faced by economic agents and/or policy makers. The inferred probabilities from the four-state MS-VAR model show evidence of a time-varying relationship. The conditional (i.e. lead-lag) and regime-prediction Granger causality provide evidence in favour of Friedman's hypothesis. This implies that past information on inflation can help improve the one-step-ahead prediction of inflation uncertainty but not vice versa. Our results have some important policy implications.
\end{abstract}

Keywords: Inflation, inflation uncertainty, seasonality, long memory, time-varying causality, Markov switching model

JEL Classification: C12,C32, E31

\section{Introduction}

Historically, inflation has been a major concern in South Africa, with inflation rates consistently within the double-digit range (Amusa et al., 2013). However, through an informal inflation targeting regime during the decade of 1990, considerable success was achieved in bringing down the inflation rate to lower levels. Given this success of informal inflation targeting, the South African Reserve Bank (SARB) decided to move to a formal inflation-targeting framework in

\footnotetext{
\# BESTMOD, Institut Supérieur de Gestion de Tunis, Université de Tunis, Tunisia. Email: adnen.bennasr@isg.rnu.tn.

\$ Department of Economics, Eastern Mediterranean University, Famagusta, Northern Cyprus, via Mersin 10, Turkey; Department of Economics, University of Pretoria, Pretoria, 0002, South Africa. Email: mehmet@ mbalcilar.net.

- College of Sciences and Humanities in Slayel, Salman bin Abdulaziz University, Kingdom of Saudi Arabia. Email: ajmi.ahdi.noomen@gmail.com.

* Department of Economics, University of Pretoria, Pretoria, 0002, South Africa. Email: goodness.aye@ gmail.com.

* Department of Economics, University of Pretoria, Pretoria, 0002, South Africa. Email: rangan.gupta@up.ac.za.

*Department of Economics, University of Pretoria, Pretoria, 0002, South Africa. Email: renee.vaneyden@up.ac.za.
} 
February 2000, with the Finance Minister declaring that the sole objective of the SARB would be to maintain inflation within a target band of 3-6\% (Mboweni, 2003; van der Merwe, 2004). The inflation rate averaged 9.5\% from 1968 to 2014, with a maximum of 20.9\% in January 1986 and a minimum of $0.2 \%$ in January 2004. As at May 2014, the inflation rate was $6.6 \%$, the highest increase since July 2009 when it stood at 6.8\% (Statistics South Africa, 2014; Trading Economics, 2014). Subsequently, due to monetary policy intervention, price increases have slowed down to $6.4 \%$ in August 2014, but still remain outside the target band of 3-6\%. Inflation and its volatility can impose costs on the real economic output, and hence on the welfare of the citizens of any nation (Chowdhoury 2014). Minimizing the adverse economic consequences and welfare costs of increases in the inflation rate would require policy makers to have a clear understanding of the major channels through which inflation may affect the real economy. This paper contributes to this understanding by considering the effects that higher inflation may have on inflation uncertainty. We also test for reverse causal effects.

The real effect of inflation was first postulated by Friedman (1977). Since then, there has been a considerable interest in the topic both theoretically and empirically with both producing conflicting results. According to Friedman's theory, there are two channels to this effect. First, an increase in the average rate of inflation may encourage unpredictable policy response by the monetary authority, thus leading to more nominal uncertainty about future inflation. Second, the growing inflation uncertainty would distort the effectiveness of the price mechanism in allocating resources efficiently, consequently causing adverse effects on real economic activities. Ball (1992) using an asymmetric information game and focusing mainly on Friedman's first channel, demonstrates that high inflation uncertainty is caused mainly by inflation, thus supporting Friedman's hypothesis. Contrary to Friedman and Ball, Pourgerani and Maskus (1987) and Ungar and Zilberfab (1993) show that the effect may actually be the opposite. While Pourgerani and Maskus (1987) show that agents may invest more resources to forecast inflation when it is rising, thus decreasing inflation uncertainty, Ungar and Zilberfab (1993) show that high inflation does not necessarily imply high inflation uncertainty.

Cukierman and Meltzer (1987) argue that high inflation uncertainty leads to high inflation levels. This emphasises a reverse causality running from inflation uncertainty to inflation. The government differ in their stabilization objectives with a trade-off between a desire to increase output through monetary surprises and a desire to keep the inflation rate low. Hence, Cukierman and Meltzer (1987) show that high inflation uncertainty may create the incentives for the monetary authority to surprise the public to have output gains by increasing the optimal average rate of inflation. However, Holland (1995) postulates that higher nominal inflation uncertainty has a negative effect on average inflation rate (i.e. increase in inflation uncertainty lowers average inflation), a hypothesis popularly known as 'stabilization Fed hypothesis'. He shows that an increase in inflation would precede or Granger cause an increase in uncertainty which would constrain the monetary authority to contract the money supply growth in order to eliminate inflation uncertainty and related negative welfare effects. This is because an increase in inflation uncertainty is perceived as costly by policy makers, thus inducing them to reduce inflation in the future.

These different hypotheses on the relationship between inflation and inflation uncertainty have given rise to a large empirical literature as will be seen in the next section. The relationship between inflation and inflation uncertainty may be sensitive to how inflation uncertainty is measured. Therefore, accurate specification of the inflation dynamics is critical. In this study we use a Seasonal Fractionally Integrated Smooth Transition Autoregressive Asymmetric Power GARCH (SEA-FISTAR-APGARCH) model to generate the conditional variance of inflation as a 
proxy for inflation uncertainty. This approach has some appealing advantages in that it is capable of handling nonlinearity, long memory and seasonal fluctuations simultaneously. It is the capability of this model that distinguishes the current study from previous studies on inflation and inflation uncertainty. In addition, unlike the standard practice in the literature, where a measure of uncertainty would be obtained based on full-sample estimation of the SEA-FISTAR-APGARCH model, we use a recursive estimation scheme to obtain a time-varying measure of uncertainty to capture for possible breaks in the parameters measuring the uncertainty - a likely event in an emerging market, but also, mimic better the real-time situation faced by agents and policy makers who would construct a measure of uncertainty based on new information and updating their previous estimates at each point in time. Further, a conditional Guassian Markov switching vector autoregression (MS-VAR) model is employed to test for causality between inflation and inflation uncertainty. The MS-VAR model is capable of handling nonlinearities in the relationship that may arise due to structural breaks or regime changes unlike the standard Granger causality test employed in most of the previous studies. Moreover, it provides information about the signs and direction of causality.

\section{Literature Review}

The empirical literature encompasses numerous approaches to estimating inflation uncertainty and testing the relationship between this and inflation. While a number of studies use the cross-sectional dispersion of individual forecasts from surveys or a moving average standard deviation of inflation as a proxy for inflation uncertainty, others have considered the nonlinear and long memory properties of inflation rates (Baillie et al., 1996a, 1996b, 2002; Hwang, 2001). In general, the results are mixed. For example, Ballie et al. (1996a) analyse the relationship between inflation and its uncertainty using maximum likelihood estimates of ARFIMA-GARCH models on monthly post World WAR II CPI data for ten countries. They find that for low inflation countries such as Canada, Germany, Italy, Japan and USA, there appear to be no relationship between the conditional mean and variance of inflation whereas for high inflation countries such as Argentina, Israel, Brazil, and UK, there is strong evidence of joint feedback between the two series.

Grier and Perry (2000) use GARCH-M methods to test the effects of real and nominal uncertainty on average inflation and output growth in the United States from 1948:07 to 1996:12. They find evidence in support of the Friedman hypothesis but find no evidence in support of the Cukierman-Meltzer hypothesis. Also, Karananos et al. (2004) examine the relationship between inflation and inflation uncertainty in the USA using a GARCH model on monthly CPI covering the period 1960:01 to 1999:02 and find strong evidence for a positive bidirectional relationship between inflation and inflation uncertainty, thus supporting both the Friedman and Cukierman-Meltzer hypotheses.

Conrad and Karanasos (2005) use long memory models (ARFIMA-FIGARCH) to generate inflation uncertainty from USA, Japan and UK CPI series for the period 1962:01 to 2001:12 and find that inflation significantly raises inflation uncertainty in line with Friedman. While increased nominal uncertainty increased inflation in Japan in line with Cukierman-Meltzer hypothesis, the impact in the UK is mixed depending on the lag structure and order of the VAR. For the USA, inflation uncertainty does not Granger cause inflation. Fountas and Karanasos (2007) use monthly data for the G7 countries, covering the period 1957-2000, and generate inflation uncertainty using a univariate GARCH model. They find mixed evidence of the Cukierman-Meltzer hypothesis but strong evidence in favour of the Friedman hypothesis, except for Germany. 
Ozdemir (2010), using a fractionally integrated vector ARMA-BEKK MGARCH model on the UK data, finds mixed evidence in the case of the Friedman hypothesis and strong evidence in favour of Cukierman-Meltzer hypothesis for the 1957:02-2006:04 period, while the evidence found following the late 1980s indicates no linkages between inflation and its uncertainty. Jiranyakul and Opiela (2010) investigates the linkage between inflation and inflation uncertainty in the ASEAN-5 countries over the period 1970:01-2007:12 with inflation uncertainty estimated as a conditional variance in an AR-EGARCH model. Their Granger causality tests show that rising inflation increases inflation uncertainty and that rising inflation uncertainty increases inflation in all five countries thus supporting both the Friedman and Cukierman-Meltzer hypotheses.

Payne (2008) uses ARMA-GARCH models to estimate inflation uncertainty and test for the relationship between inflation and inflation uncertainty using Granger causality tests for three Caribbean countries: the Bahamas, Barbados, and Jamaica. The results indicate that an increase in inflation leads to an increase in inflation uncertainty for the Bahamas and Barbados, supporting the Friedman-Ball hypothesis. However, an increase in inflation uncertainty leads to a decrease in inflation in the case of Jamaica thus supporting Holland's stabilization-motive hypothesis.

In a study on the G3 countries (USA, Japan and UK), Balicilar, Ozdemir and Cakan (2011) use GARCH to generate inflation uncertainty from monthly CPI series for the period 1957:01 to 2006:10 and linear and nonlinear Granger causality tests to examine the causal relationship between inflation and its uncertainty. Results from both parametric and non-parametric tests support both the Friedman and Cukierman-Meltzer hypotheses in all countries meaning that inflation and inflation uncertainty have predictive content for each other.

Chen, Shen and Xie (2008) examine the Friedman and Cukierman-Meltzer hypotheses for four economies in East Asia, namely Taiwan, Hong Kong, Singapore, and South Korea, using both linear and nonlinear flexible regression models. Using moving average standard deviation as a proxy for inflation uncertainty, their findings show that for the linear model, the Friedman hypothesis is rejected for all four economies. The nonlinear model supports the Friedman hypothesis for all economies except Hong Kong. Inferences from the nonlinear model support the Cukierman-Meltzer hypothesis for all economies, with Hong Kong, Singapore and South Korea showing the positive effect of inflation uncertainty on inflation while Taiwan exhibit an inverted-U shaped relationship. The linear model rejects the Cukierman-Meltzer hypothesis for Hong Kong and Singapore.

Balcilar and Ozdemir (2013a) examine the asymmetric and time-varying causality between inflation and inflation uncertainty using data for the G7 countries, covering the period 1959:12 to 2008:10. Inflation uncertainty is generated from a Fractionally Integrated Smooth Transition Autoregressive Moving Average Asymmetric Power ARCH (FISTARMA-APARCH) model which captures both the nonlinear and long memory features. Using a conditional Guassian Markov switching vector autoregression (MS-VAR) model to test for causality, they find strong evidence in favour of the Holland's 'stabilization Fed hypothesis' for Canada, France, Germany, Japan, UK, and USA, while the Friedman hypothesis is supported in Canada and USA.

Chowdhury (2014) investigates the relationship between inflation and inflation uncertainty for India using Granger causality tests. He uses a GARCH model for estimating inflation uncertainty from monthly wholesale price index (WPI) from 1954:04 to 2010:04. The maximum likelihood estimate of the GARCH model provides strong evidence of a positive relationship between inflation and inflation uncertainty while the causality test indicates a bidirectional causality between the two series.

Other recent international literature on inflation and inflation uncertainty includes Ozdemir and Fisunoğlu (2008) on Jordan, Philippines and Turkey, Coporale and Kontonikas (2009) on 12 
EMU countries, Hasanov and Omay (2011) on selected CEE countries, Karahan (2012) on Turkey, Viorica et al. (2014) on the newest EU countries.

As far as Africa is concerned, there is a dearth of studies on the relationship between inflation and inflation uncertainty. The only known studies are those of Thornton (2007), Valdovinos and Gerling (2011), Hegerty (2012), Barimah and Amuakwa-Mensah (2012), Pretorius (2012), Oteng-Abayie and Doe (2013) and Barimah (2014). Thornton (2007) employs GARCH models to construct the variance of inflation as a measure of monthly inflation uncertainty in 12 emerging market economies, which includes South Africa, and examine the direction of causality between inflation and inflation uncertainty using standard Granger causality tests. In general, he finds stronger support for the Friedman hypothesis that higher inflation rates raise inflation uncertainty, but the results concerning the effect of inflation uncertainty on average inflation are mixed. For South Africa specifically, he finds evidence of inflation Granger causing inflation uncertainty but not vice versa.

Valdovinos and Gerling (2011) examine the relationship for eight member countries of the West African Economic and Monetary Union (WAEMU), namely Benin, Burkina Faso, Côte d'Ivoire, Guinea-Bissau, Mali, Niger, Senegal and Togo, using monthly CPI data covering 1994 to 2009. They obtain the time-varying conditional variance of the error term as the measure of inflation uncertainty for each country based on a GARCH model. The Granger causality test results support the Friedman-Ball hypothesis for all WAEMU countries. They also find that the overall effect of inflation on inflation uncertainty is positive, as the sum of the coefficients on lagged inflation is positive. However, they also find some evidence for the Cukierman hypothesis, as the null hypothesis that inflation uncertainty does not Granger cause inflation is rejected for Benin, Senegal, and Togo.

Hegerty (2012) employs exponential GARCH (EGARCH and EGARCH-M) models to measure inflation uncertainty from monthly CPI covering January 1976 to late 2011 or 2012 for 9 sub-Saharan African countries, including Bukina Faso, Botswana, Côte d'Ivoire, Ethiopia, Gambia, Kenya, Nigeria, Niger and South Africa. Subsequently the relationship between inflation and its uncertainty for each country is investigated using Granger causality tests and impulse response functions. The impulse responses show that shocks to inflation lead to increases in inflation uncertainty in all cases while the responses of inflation to inflation uncertainty was more ambiguous. The Granger causality test also supports the Friedman hypothesis for all countries as inflation causes inflation uncertainty; the reverse is, however, not true for half of the countries including South Africa. Also the multi-country Granger causality tests shows that regional spill-overs occur mainly among Western African neighbours.

Using inflation uncertainty data generated from $\operatorname{GARCH}(1,2)-\mathrm{M}$ type models, Barimah and Amuakwa-Mensah (2012) examine the relationship between inflation and inflation uncertainty in Ghana from 1964:04 to 2012:12. The results from the Granger causality tests show that inflation significantly raises inflation uncertainty over the full sample and two subsamples at different lag lengths, thus supporting the Friedman-Ball hypothesis. Further, they find that inflation uncertainty affect inflation as suggested by Cukierman-Meltzer hypothesis only in the long run (i.e. at lags exceeding 7 months). In a related study on Ghana, Oteng-Abayie and Doe (2013) use a GARCH model and Granger causality tests to investigate the relationship for the period 1984 to 2011. They find that inflation uncertainty rises in the high inflationary periods and that inflation uncertainty Granger causes inflation in support of the Cukierman-Meltzer hypothesis. Employing a Full Information Maximum Likelihood (FIML) technique to estimate an alternative GARCH-M model, they find that inflation uncertainty in the inflation equation is significant - thus confirming the Granger causality results that higher inflation uncertainty raises inflation. 
Pretorius (2012) examines the relationship between inflation, inflation uncertainty and economic growth in South Africa using quaterly data for the period 1960:01 to 2012:01. Inflation uncertainty is estimated from a GARCH-M model for the period. The Granger causality test indicates that inflation uncertainty has a negative impact on inflation, thus supporting Holland's hypothesis of stabilizing central bank's behaviour. Conversely, he find evidence that high inflation leads to increased inflation uncertainty in line with Friedman's hypothesis.

Barimah (2014) investigates the relationship for Ghana using more updated monthly CPI data (1963:4 to 2014:2) and different methods (AR-EGARCH models) than used in Barimah and Amuakwa-Mensah (2012) to generate inflation uncertainty. Including an inflation dummy in a variance equation and also using Granger cauality tests, he finds strong support for both Friedman-Ball and Cukierman-Meltzer hypotheses for both the full sample and the inflation targeting period.

From the foregoing, it is very clear that the inflation and inflation uncertainty hypotheses have not been adequately examined for African countries in general and South Africa in particular. Therefore, the current study aims at examining the dynamic relationship between inflation and inflation uncertainty in South Africa. This issue is particularly important in South Africa given the recent surge in inflation. We contribute by employing a recursively estimated Seasonal Fractionally Integrated Smooth Transition Autoregressive Asymmetric Power GARCH (SEA-FISTAR-APGARCH) model to generate the conditional variance of inflation as a proxy for inflation uncertainty, thereby accounting for the nonlinear, long memory and seasonal features of inflation simultaneously. Subsequently, we follow Balcilar and Ozdemir (2013a, 2013b) and test Granger causality using a conditional Gaussian Markov switching vector autoregressive (MS-VAR) model. The MS-VAR model is not only capable of handling nonlinearities in the relationship that may arise due to structural breaks or regime changes unlike the standard Granger causality test employed in most of the previous studies, but also, it provides information about the signs, along with the direction of causality. So our paper, not only improves the South African literature on inflation uncertainty and inflation technically, but also analysing the causal relationship over the longest possible sample of inflation data available, provides us to track the causality historically between these two variables.

\section{Methodology}

\subsection{Estimation of Inflation Uncertainty}

\subsubsection{The Seasonal FISTAR-APGARCH model}

A large number of possible specifications are available incorporating many stylized features of economic time series. Empirical studies by Glosten et al. (1989), Nelson (1991), and Engle and $\mathrm{Ng}$ (1992) show that it is crucial to capture asymmetry in economic time series models. Ball and Mankiw (1994) show that sticky prices with a positive trend in inflation imply asymmetric (nonlinear) inflation adjustment, because forward-looking firms setting their prices for several periods will incorporate the effects of the inflation. Enders and Hurn (2002) also obtain evidence of asymmetric inflation adjustment. The seasonal FISTAR model (SEA-FISTAR) proposed by Ajmi et al. (2008) is able to capture nonlinearity, long memory and seasonal fluctuations 
simultaneously. The SEA-FISTAR model represent an extension of the FISTAR model introduced by Van Dijk et al. (2002) by allowing for an explicit description of the seasonal pattern of the series, i.e. using seasonal dummy variables.

The SEA-FISTAR model is given by:

$$
(1-L)^{d} y_{t}=x_{t}
$$

with

$$
\begin{aligned}
x_{t}= & \left(\sum_{s=1}^{S} D_{s, t} \mu_{1, s}\right)\left(1-F\left(S_{F, t} ; \gamma_{F}, c_{F}\right)\right)+\left(\sum_{s=1}^{S} D_{s, t} \mu_{2, s}\right) \times F\left(S_{F, t} ; \gamma_{F}, c_{F}\right) \\
& +\left(\sum_{i=1}^{p} \phi_{1, i} x_{t-i}\right)\left(1-G\left(S_{G, t} ; \gamma_{G}, c_{G}\right)\right)+\left(\sum_{i=1}^{p} \phi_{2, i} x_{t-i}\right) \times G\left(S_{G, t} ; \gamma_{G}, c_{G}\right)+\varepsilon_{t}
\end{aligned}
$$

$d$ is the fractional integration order of the process (see Granger and Joyeux, 1980). $L$ is the backshift operator such that $L y_{t}=y_{t-1} . D_{s, t}$ are the seasonal dummy variables with $D_{s, t}=1$ in season $s, 0$ elsewhere. $S$ is the number of seasons in one year, $\mu_{j, s}$ are the seasonal means in regime $j, j=1,2 . \phi_{j, i}, i=1, \ldots, p$ are the autoregressive parameters in regimej. $\varepsilon_{t} \sim$ i.i.d. $\left(0, \sigma_{\varepsilon}^{2}\right), F\left(S_{F, t} ; \gamma_{F}, c_{F}\right)$ and $G\left(S_{G, t} ; \gamma_{G}, c_{G}\right)$ are two logistic transition functions which are defined as (see van Dijk et al. (2002)):

$$
F\left(S_{F, t} ; \gamma_{F}, c_{F}\right)=\left[1+\exp \left(-\frac{\gamma_{F}}{\sigma_{S_{F, t}}}\left(S_{F, t}-c_{F}\right)\right)\right]^{-1}
$$

$S_{F, t}$ is the transition variable of the transition function $F \cdot \gamma_{F}$ is the parameter which determines the smoothness of the function $F . \sigma_{S_{F, t}}$ is the standard deviation of the transition variable $S_{F, t}$. Finally, $c_{F}$ is the threshold of the transition from one regime to another. The second transition function $G\left(S_{G, t} ; \gamma_{G}, c_{G}\right)$ is described in the same way.

Equations (1)-(2) are the general representation of the seasonal FISTAR model. However, some particular cases can be discussed according to the variables and the parameter values of the transition functions. First, when $\gamma_{F}=\gamma_{G}=0$, equation (2) reduces to a linear seasonal autoregressive model. Second, if $\gamma_{F}=0$, then $F\left(S_{F, t} ; \gamma_{F}, c_{F}\right)=\frac{1}{2}$ for all values of $S_{F, t}$ and only the autoregressive parameters change between regimes. There is no regime switching in the seasonal component. Third, when $F\left(S_{F, t} ; \gamma_{F}, c_{F}\right)=G\left(S_{G, t} ; \gamma_{G}, c_{G}\right)$, the seasonal FISTAR model is restricted in a way that both seasonal and autoregressive parameters change simultaneously, and with the same speed of transition from $\mu_{1, s}$ and $\phi_{1, i}$ to $\mu_{2, s}$ and $\phi_{2, i}$ respectively, $s=1, \ldots, S$ and $i=1, \ldots, p$. $^{1}$

Ajmi et al. (2008) introduced an empirical specification of the Seasonal FISTAR model using the "specific to general" procedure (see Granger, 1993). To this aim, Ajmi et al. (2008) extend the specification procedure elaborated by Teräsvirta (1994) for STAR models and van Dijk et al. (2002) for FISTAR models to specify the empirical representation of the seasonal FISTAR model in equations (1)-(2).

${ }^{1}$ For more details, see Ajmi et al. (2008). 
We obtain the inflation uncertainty by allowing $\varepsilon_{t}$ in equation (2) to follow a GARCH process. Parametric GARCH models have emerged as the archetype for modelling time-varying and predictable volatility.

Baillie (2002) shows that the long memory not only exists in the mean, it also exists in the second moment of inflation rates. He finds that the squared and absolute values of inflation residuals estimated using a fractional filter applied to the conditional mean, also have memory properties. Therefore, conditional variance of inflation rates should possibly be modelled as a long memory autoregressive conditional heteroscedastic process. One such model is the asymmetric power GARCH model (APGARCH) proposed by Ding et al. (1993). The APGARCH had considerable success in modelling time-varying conditional heteroskedasticity with asymmetry. Furthermore the simulated autocorrelation function mirrors the long memory features. The general structure is as follows:

$$
\begin{aligned}
{ }_{t} & =h_{t} v_{t}, \quad{ }_{t} \sim N(0,1) \\
h_{t} & =+{ }_{i=1}^{n}\left(||_{t} i \mid{ }_{i} t_{i}\right)+{ }_{i=1}^{r} i_{i} t_{i}
\end{aligned}
$$

where $\delta>0$ plays the role of a Box-Cox transformation of the conditional standard deviation $h_{t}$, while the $\theta_{i}$ 's reflect the so-called leverage effect. A positive (negative) value of the $\theta_{i}$ 's means that past negative (positive) shocks have a stronger impact on current conditional volatility than past positive shocks (see Black, 1976).

For a given data series, our empirical specification consists of the following steps:

1. Specify the autoregressive order $p$ for the adequate seasonal ARFI model using the BIC criterion.

2. Test the null hypothesis of linearity against seasonal FISTAR model and select the appropriate transition function.

3. Estimate the seasonal FISTAR model.

4. Evaluate the model using diagnostic tests.

\section{Nonlinearity test}

The null hypothesis of linearity holds if the seasonal and the autoregressive parameters are constant over the different regimes, i.e., $H_{0}: \mu_{1, s}=\mu_{2, s}$ and $\phi_{1, i}=\phi_{2, i}, i=1, \ldots, p$ and $s=1, \ldots, S$. This null hypothesis can be expressed in another way: $H_{0}^{\prime}: \gamma_{F}=\gamma_{G}=0$. The alternative hypothesis of nonlinearity is given by $H_{1}: \mu_{1, s} \neq \mu_{2, s}$ and/or $\phi_{1, i} \neq \phi_{2, i}$, for at least one value of $i, s$. However, under the null hypothesis $H_{0}^{\prime}$, the model is not identified. To resolve this problem, we follow the approach of Luukkonen et al. (1988) by replacing the transition functions by their first order Taylor expansion with respect to $\gamma$ around 0 .

This null hypothesis of linearity, for general representation equations (1)-(2), can be tested using a Lagrange Multiplier $\left[L M_{3}\right]$ statistic by following the next stages: 
1. Estimate the seasonal ARFI model and compute the residuals $\hat{\varepsilon}_{t}$ and the sum of squared residuals $S S R_{0}=\sum_{j=1}^{T} \hat{\varepsilon}_{t}^{2}$.

2. Regress $\hat{\varepsilon}_{t}$ on $D_{t} S_{F, t}^{i}, w_{t} S_{G, t}^{i}$ and $-\sum_{j=1}^{t-1} \frac{\hat{\varepsilon}_{t-j}}{j}, i=0,1,2,3$; and compute the sum of squared residuals from this regression, $S S R_{1}$.

3. The $L M_{3}$ statistic is given by

$$
L M_{3}=\frac{S S R_{0}-S S R_{1}}{\frac{S S R_{1}}{T}} \hat{\mathrm{I}} \chi^{2}(3(p+S))
$$

The nonlinearity LM test for particular cases can be performed as for the general model. Once the null hypothesis is rejected, we select the most appropriate transition variable based on the LM statistic values. That is, we select the one with the smallest $p$-value.

\section{Estimation of the SEA-FISTAR-APGARCH Model}

When the transition variable is selected from the nonlinearity test, we can estimate the seasonal FISTAR model using Beran's (1995) approximate maximum likelihood estimator for the ARFIMA model which was adapted by van Dijk et al. (2002) to estimate the FISTAR model. The estimated parameters $\hat{\theta}$ are obtained by minimizing the sum of squared residuals:

$$
Q_{T}(\theta)=\sum_{t=1}^{T} e_{t}^{2}(\theta)
$$

where $\theta=\left(\mu_{1}^{\prime}, \mu_{2}^{\prime}, \phi_{1}^{\prime}, \phi_{2}^{\prime}, \gamma_{F}, \gamma_{G}, c_{F}, c_{G}, d\right)^{\prime}, \mu_{j}=\left(\mu_{j, 1}, \ldots, \mu_{j, S}\right)^{\prime}$ and $\phi_{j}=\left(\phi_{j, 1}, \ldots, \phi_{j, p}\right)^{\prime}, j=$ 1,2. Starting values needed in the optimization algorithm can be obtained using five dimensional grid search over $d, \gamma_{F}, \gamma_{G}, c_{F}$ and $c_{G}$. The selected starting values are those that give the smallest estimator for the residuals variance $\hat{\sigma}^{2}\left(\gamma_{F}, \gamma_{G}, c_{F}, c_{G}, d\right)$. For the cases when $\gamma_{F}=0$ or when $\left.F\left(S_{F, t} ; \gamma_{F}, c_{F}\right)\right)=G\left(S_{G, t} ; \gamma_{G}, c_{G}\right)$, the estimation procedure is performed in a straightforward manner.

For simplicity, we adopt a two-step estimation procedure for the SEA-FISTAR-APGARCH model. We first estimate the Seasonal FISTAR parameters in equations (1)-(3); subsequently the parameters of the APGARCH part in equation (4) are estimated based on the residuals of the Seasonal FISTAR part. However, unlike the standard practice in the literature where the full-sample is used to obtain a measure of uncertainty, we undertake a recursive estimation of the SEA-FISTAR-APGARCH model to mimic the real-time situation faced by an agent and/or policymakers for measuring uncertainty. Specifically, we first estimate the SEA-FISTAR model recursively and obtain the recursively generated residuals, which in turn are used to estimate recursively the AGARCH model, to obtain a recursive measure of uncertainty. In other words, our recursive approach allows us to obtain a time-varying measure of uncertainty, with the estimate of the same being updated as new data comes in on inflation. Ideally, one would need a real-time data set with all the vintages of inflation; however given the length of the period under investigation, no such data is available. 


\section{Misspecification Test}

To evaluate the fitted seasonal FISTAR model, it is required to perform some diagnostic tests on the resulting residuals, specifically testing the residuals for serial correlation. To this aim, Ajmi et al. (2008) extended the LM test for STAR model residual serial correlation proposed by Eitrheim and Teräsvirta (1996). The null hypothesis of no residual serial correlation for seasonal FISTAR model can be tested against the alternative of serial dependence up to order $q$, given by

$$
\varepsilon_{t}=\left(\sum_{j=1}^{q} a_{j} L^{j}\right) \varepsilon_{t}+v_{t}
$$

with $v_{t} \sim i i d\left(0, \sigma^{2}\right)$ and $L$ the lag operator. The hypothesis of serial independence of $\varepsilon_{t}$, $H_{0}: a_{1}=a_{2}=\ldots=a_{q}=0$ can be tested by an $L M$ test (see Eirtheim and Teräsvirta, 1996). The no residual correlation LM test for STAR model was modified by including the gradients of $v_{t}$ with respect to the seasonal parameters, the parameters of transition function $F\left(S_{F, t} ; \gamma_{F}, c_{F}\right)$ and the fractional differencing parameter $d$.For the cases when $\gamma_{F}=0$ or when $\left.F\left(S_{F, t} ; \gamma_{F}, c_{F}\right)\right)=$ $G\left(S_{G, t} ; \gamma_{G}, c_{G}\right)$, the no residual serial correlation LM test can be modified in a straightforward manner.

\subsection{Asymmetric and Time-varying Granger Causality}

The starting point of this paper is not to assume a permanent causal relation between inflation and its uncertainty, but rather adopt a notion of 'temporary' Granger causality, that is causality that holds during some periods but not in others following Balcilar and Ozdemir (2013a, 2013b). Previous researchers have noted that results from Granger causality tests tend to be sensitive with respect to changes in the sample period ${ }^{2}$. They have also noted that the direction of causality is sensitive to the choice of the sample period. Further, there are periods with no causality along with periods with bidirectional causality between inflation and inflation uncertainty. Based on these findings, our strategy then consists of identifying the periods during which a particular type of causality holds, i.e., a period in which inflation Granger causes inflation uncertainty and vice versa. This approach also allows us to identify the periods of causality, bidirectional causality, and non-causality. The methodology we adopt is based on a VAR model with time-varying parameters where, given our objectives, parameter time-variation directly reflects changes in causality. In this approach, changes in causality are treated as random events governed by an exogenous Markov process, leading to the MS-VAR model. In the MS-VAR model inferences about changes in the causality can be made on the basis of the estimated probability that each observation in the sample comes from a particular regime of causality.

To be concrete, let $\pi_{t}$ and $h_{t}$ represent inflation rate and inflation uncertainty, respectively, and let the time series of these variables up to and including period $t$ be given by $\mathfrak{J}_{t} \equiv\{h: \tau=t$, $t-1, \ldots, 1-p\}$ and $\mathfrak{R}_{t} \equiv\{: \tau=t, t-1, \ldots, 1-p\}$, where $p$ is a nonnegative integer. Define the vector of time series of $X_{t}$ up to and including period $t$ as $X_{t}=\left[\pi_{t}^{\prime}, h_{t}^{\prime}\right]^{\prime}$ and let $\aleph_{t} \equiv\left\{\mathfrak{I}_{t}, \mathfrak{R}_{t}\right\}$, correspondingly. For the vector valued time series $X_{t}$ of random variables assume that there

\footnotetext{
${ }^{2}$ Several studies (Okun, 1971; Kontinokas, 2004; Grier and Ye, 2007) noted that the inflation-inflation uncertainty relationship is not stable. Okun (1971) argues that the relationship between inflation and inflation variability was much weaker during the 1960s. Using breakpoint tests, Kontinokas (2004) concludes that the relationship breaks around 1992 due to the introduction of inflation targeting in several countries. Using structural stability tests, Grier and Ye (2007) identifies 2 to3 structural breaks.
} 
exists a probability density function $f\left(X_{t} \aleph_{t-1}, \theta\right)$ for each $t \in\{1,2, \ldots, T\}$. The parameters and the parameter space are denoted by $\theta$ and $\Theta$, respectively. The true value of $\theta$ is denoted by $\theta_{0} \in \Theta$.

A Markov switching vector autoregressive (MS-VAR) model for inflation rate and inflation uncertainty which is nested within the class of autoregressive models is studied by Hamilton (1990) and Krishnamurthy and Rydén (1998) and allows asymmetric (regime dependent) inference for causality. The structure of the MS-VAR model we use is analogues to the one used in Warne (2000). However, our model is more general and allows inference on all types of causality relationships ${ }^{3}$. Our analysis is based on the following MS-VAR model:

$$
X_{t}=\mu_{S_{t}} D_{t}+\sum_{k=1}^{p} \Phi_{S_{t}}^{(k)} X_{t-1}+\varepsilon_{t}
$$

where $p$ is the order of the MS-VAR model, $\varepsilon_{t} \mid S_{t} \sim N\left(0, \Omega_{S_{t}}\right)$ and $\Omega_{S_{t}}$ is positive definite. The vector $D_{t}$ is $d$ dimensional and deterministic, e.g. a constant, centred seasonal dummies, and other dummies corresponding to outliers. The random state or regime variable $S_{t}$ is unobserved, conditional on $S_{t-1}$ independent of past $X$, and assumed to follow a 4-state Markov process. In other words, $\operatorname{Pr}\left[S_{t}=j \mid S_{t 1}=i, S_{t_{2}}=h_{2}, \ldots,{ }_{t 1}\right]=p_{i j}$, for all $t$ and $h_{l}, i, j=1,2, \ldots, q, l \geq 2$. In our particular application, the maintained hypothesis is that $q=4$, that is four states or regimes for each variable are sufficient to describe the causality between inflation and inflation uncertainty. For instance in the first state one variable (say inflation) may be causal for the other variable (inflation uncertainty). Analogously, in the second state that variable is not causal for the other variable, etc. When $q=4, S_{t}$ is a 4 -state Markov process, i.e. for both variables the MS-VAR model does indeed allow four types of regimes in terms of causality classification ${ }^{4}$. That is, the 4-state MS-VAR defined in equation (8) accommodates four causality results, that $\pi_{t}$ Granger causing $h_{t}\left(\pi_{t} \Rightarrow h_{t}\right), h_{t}$ Granger causing $\pi_{t}\left(h_{t} \Rightarrow \pi_{t}\right), \pi_{t}$ and $h_{t}$ both Granger causing each other (a feedback system or bidirectional causality) $\left(\pi_{t} \Leftrightarrow h_{t}\right)$, and no Granger causality between $\pi_{t}$ and $h_{t}\left(\pi_{t}<\neq>h_{t}\right)$. How these four regimes are inferred will be explained below.

The Markov transition probabilities satisfy $\sum_{j=1}^{4} p_{i j}=1$ for all $i$. It is also assumed that the Markov process is irreducible (no absorbing states) with parameters

\footnotetext{
${ }^{3}$ Warne's (2000) model is a restricted version of our model and allows testing for conditional Granger causality assuming that the regime of one of the series is given. Therefore, Warne tests whether a variable does not Granger cause another variable conditional on the known regime. Our model allows Granger causality testing without conditioning on a known regime for a variable, although it is possible to restrict the model on a given regime and obtain the tests in Warne (2000).

${ }^{4}$ An MS-VAR model in which each of two variables following two independent states has a total of four inferred states. Such an MS-VAR model can be reformulated in terms of a latent state variable with four states, where each variable follows a separate and independent 2-state Markov process. This 4-state classification allows an intuitive interpretation of the four possible causality regimes. We later reformulate the MS-VAR model in a 4-state form using two independent Markov processes for a clear representation of the causal classification between inflation and its uncertainty series. Indeed, each variable follows two states, which may characterize expansions and contractions. Our representation flexibly does not restrict both variables to the same regime at any point in time.
} 


$$
P=\left[\begin{array}{ccc}
p_{11} & \cdots & p_{14} \\
& \vdots & \\
p_{41} & & p_{44}
\end{array}\right]
$$

One eigenvalue of $P$ is always equal to unity by construction and to ensure that $S_{t}$ is ergodic, the remaining eigenvalues are assumed to lie inside the unit circle. The ergodic probabilities, $\operatorname{Pr}\left[S_{t}=j\right]=\pi_{j}$, are collected into $\lambda$, where $P^{\prime} \lambda=\lambda$. The ergodicity of $S_{t}$ guarantees the ergodicity of $\mu_{S_{t}}, \Phi_{S_{t}}^{(k)}$, and $\Omega_{S_{t}}$. The parameters of the MS-VAR model, $\mu_{S_{t}}, \Phi_{S_{t}}^{(k)}$, and $\Omega_{S_{t}}$ in equation (8) depend only on the regime variable $S_{t}$. That is, if $S_{t}=j$, then $\mu_{S_{t}}=\mu_{j}$, $\Phi_{S_{t}}^{(k)}=\Phi_{j}^{(k)}$ and $\Omega_{S_{t}}=\Omega_{j}$.

We assume that the two subsystems, one in which $\pi_{t}$ Granger causing $h_{t}$ and the other in which $h_{t}$ Granger causing $\pi_{t}$, of the Granger causality regimes are independent. This implies coefficients in two subsystems of equation (8) vary with the regime and, at the same time are independent. A Markov chain that splits into two independent processes each with two regimes will have this property.

Let $S_{1, t}$ and $S_{2, t}$ be a $q_{1}=2$ and a $q_{2}=2$ state Markov process, respectively, with $q=$ $q_{1} q_{2}=4$ and $S_{1, t}$ and $S_{2, t}$ independent, i.e. $p_{i j}=p_{i_{1} j_{1}}^{(1)} p_{i_{2} j_{2}}^{(2)}$ where $\operatorname{Pr}\left[S_{l, t}=j_{l} \mid S_{l, t-1}=i_{l}\right]=p_{i_{l} j_{l}}^{(l)} \quad$ and $\sum_{j_{l}=1}^{q_{l}} p_{i_{l} j_{l}}^{(l)}=1$ for $i_{l}=1, \ldots, q_{l}$. Collecting the probabilities of two states into $P^{(1)}$ and $P^{(2)}$ and defining $S_{t}=S_{2, t}+q_{2}\left(S_{1, t}-1\right)$ for the pair $\left(S_{1, t}, S_{2, t}\right)$ we have that $P=\left(P^{(1)} \otimes P^{(2)}\right)$. Thus, $S_{1, t}$ and $S_{2, t}$ are latent random variables that reflect the state or regime of the system at date $t$ and take their values in the set $\{1,2\}$. Two independent subsystems do indeed define four alternative states of the nature ${ }^{5}$, which are conveniently indexed by $S_{t}$ as

$$
S_{t}=\left\{\begin{array}{cc}
1, & \text { if } S_{1, t}=1 \text { and } S_{2, t}=1 \\
2, & \text { if } S_{1, t}=2 \text { and } S_{2, t}=1 \\
3, & \text { if } S_{1, t}=1 \text { and } S_{2, t}=2 \\
4, & \text { if } S_{1, t}=2 \text { and } S_{2, t}=2
\end{array}\right.
$$

Using the above definition of the MS-VAR model we can link inference about the Markov regime with Granger causality. Consider the MS-VAR in equation (8) for $X_{t}=\left[\pi_{t}^{\prime}, h_{t}^{\prime}\right]^{\prime}$ :

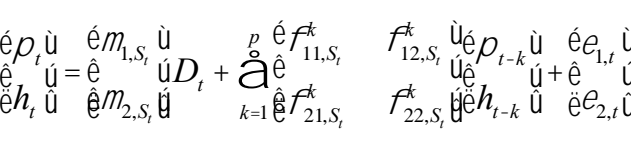

\footnotetext{
${ }^{5}$ These four states correspond four types of causality regimes relating to our model defined in equation (8). Defining these regimes in terms of two independent states gives us the flexibility of fixing one of the variables in a particular state while allowing the other variable to follow two states.
} 
Here $\varepsilon_{t}=\left[\varepsilon_{1, t}^{\prime}, \varepsilon_{2, t}^{\prime}\right]^{\prime}$ is a white noise process, independent of $S_{t}$ with mean zero and covariance matrix $\Omega_{S_{t}}$, partitioned conformably with $\varepsilon_{i, t}$, i.e., $\Omega_{i j, S_{t}}=E\left[\varepsilon_{i, t}^{\prime} \varepsilon_{j, t} \mid S_{t}\right]$.

Let $\varepsilon_{1, t+1}$ denote the 1-step ahead forecast error for one-period-ahead inflation $\pi_{t+1}$ conditional on $\aleph_{t}$ and the known parameter vector $\theta_{0}$ when the predictor is given by the expectations operator, $E$. That is,

$$
\varepsilon_{1, t+1}=\pi_{t+1}-E\left[\pi_{t+1} \mid \aleph_{t}, \theta_{0}\right]
$$

$\varepsilon_{1, t+1}$ satisfies the usual assumptions, that it has a zero conditional mean and positive finite conditional variance $\sigma_{t}^{2}$. The causality defined in Granger (1969) concerns the optimal (minimum MSE) unbiased 1-step ahead linear least squares predictor and cannot be directly applied to nonlinear models. For our purpose it is sufficient to adopt a generalization of Granger causality, which is applicable to nonlinear models (see Warne, 2000). Inflation uncertainty is said to be noncausal (in the mean) for inflation if and only if for all $t$

$$
E\left[\varepsilon_{1, t+1}^{2} \mid \theta_{0}\right]=E\left[\tilde{\varepsilon}_{1, t+1}^{2} \mid \theta_{0}\right]<\infty
$$

where $\widetilde{\varepsilon}_{1, t+1}^{2}=\pi_{t+1}-E\left[\pi_{t+1} \mid \Re_{t}, \theta_{0}\right]^{6}$. An analogous definition could be given for the noncausality of inflation for inflation uncertainty. The definition in equation (13) may hold in two instances: firstly when the Markov process is serially uncorrelated, that is $\operatorname{Pr}\left[S_{t+1} \mid S_{t}\right]=\operatorname{Pr}\left[S_{t+1}\right]$; and secondly when $\operatorname{Pr}\left[S_{t} \aleph_{t}\right]=\operatorname{Pr}\left[S_{t} \mid \Re_{t}\right]$. These two cases exemplify the two channels in an MS-VAR model through which the inflation uncertainty can Granger cause inflation and vice versa.

The first channel leads to the regime-prediction Granger causality test, which investigates whether the shocks to the inflation uncertainty is helpful in predicting the regime of the inflation (an indirect prediction channel). In this case inflation uncertainty becomes useful in predicting the inflation. The second channel leads to the conditional Granger causality test, which investigates the lead-lag relationship assuming the regime is known. This test is conditional on the known regime, and therefore, it is a test of whether inflation uncertainty helps improve the one-step-ahead forecast of inflation. For instance, if the regime is known and inflation uncertainty cannot help to improve the one-step-ahead prediction of inflation, then inflation uncertainty does not Granger cause inflation conditional on the given regime. This test is analogous to the traditional Granger causality test conditional on the given regime. If either of the conditional Granger noncausality or regime-prediction Granger noncausality is rejected, the null hypothesis of Granger noncausality will also be rejected.

This definition of Granger noncausality of inflation uncertainty for inflation given in equation (13) presumes that the coefficients in the inflation equation vary freely with the regime. It is possible, however, that these coefficients vary with $S_{1, t+1}$ but not with $S_{2, t+1}$. There may be

\footnotetext{
${ }^{6}$ It is quite straightforward to extend this definition to allow for causality in the variance as well as in the mean. Granger et al. (1986) gives the definition of causality in mean-variance in a linear model. The Granger noncausality in mean-variance can be stated as $E\left[\varepsilon_{1, t+1}^{2} \mid \theta_{0}, \aleph_{t}\right]=E\left[\tilde{\varepsilon}_{1, t+1}^{2} \mid \theta_{0}, \Re\right]<\infty$.
} 
information in the past inflation uncertainty, $\mathfrak{J}_{t}$, for predicting $S_{2, t+1}$ but not for predicting $S_{1, t+1}$. The prediction of inflation obtained from equation (11) may still not depend on the history of inflation uncertainty. Thus a formal and operational definition of Granger noncausality incorporating these considerations in a nonlinear model should be given ${ }^{7}$. Conditions for Granger noncausality are not straightforward, because there are two channels through which inflation uncertainty, for example, can be informative about inflation. Therefore, no unique set of parameter restrictions can be specified for testing the noncausality of inflation uncertainty for inflation, and vice versa. However, there is a finite number of cases and if one of these cases is true, then the inflation uncertainty, for example, is Granger noncausal (in mean-variance) for inflation ${ }^{8}$.

We now give the conditions under which equation (13) holds within the context of the bivariate MS-VAR model in equation (11), that is, the conditions under which the regime prediction Granger causality is violated. Inflation uncertainty is noncausal for inflation, that is the regime forecasts $S_{1, t+1}$ and $S_{2, t+1}$ are independent and there is no information in $\mathfrak{J}_{t}$ for predicting $S_{1, t+1}$, if and only if for all $t$

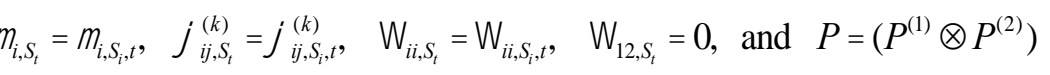

for all $i, j=1,2, k=1, \ldots, p$, and $S_{t}=1, \ldots, 4 ;$ and

$$
\phi_{12}^{(k)}=0
$$

for $k=1, \ldots, p$, and $S_{1, t}=1,2$ (see Warne, 2000) ${ }^{9}$. The condition given in equation (15) is straightforward to interpret. For expositional purpose let us assume that all regimes are known. Then, the necessary and sufficient condition for inflation uncertainty to Granger cause inflation (in mean-variance, and also in distribution ${ }^{10}$ ) is that $\phi_{12}^{(k)}$ in equation (11) is equal to zero for all $k$ and $t$. When the regimes are unknown then the additional restrictions in equation (14) should also hold for noncausality of inflation uncertainty. These noncausality conditions are given as the null hypothesis $H_{0}^{1}$ in Table 1. The 4-state MS-VAR specification with conditions in (14) and (15) is the most general case and allows separation of four types of regime classifications. In this general case, in order for the inflation uncertainty to be noncausal for inflation, all coefficients in the inflation equation must be constant, while the coefficients on lags of inflation uncertainty are all zero; and additionally all error variances should be constant and covariance across the error terms should be zero. Note also that all restrictions in equations (14) and (15) are linear. If we change the restrictions in equation (15) to $\phi_{21}^{(k)}=0$ and change equation (14) accordingly, then there is

\footnotetext{
${ }^{7}$ The Granger noncausality condition given in equation (13) cannot be directly tested and one needs to derive the conditions that need to be imposed on the parameters of the model under which equation (13) holds. In an MS-VAR model implied restrictions involve all parameters in equation (8) as well as restrictions on the covariance matrix $S_{t}$ and transition probabilities $P$.

${ }^{8}$ Jacobson et al. (2002) consider a 2-state MS-VAR model with three variables and lists four conditions for a variable to be noncausal.

${ }^{9}$ Warne (2000) proposes conditions under which one variable is not Granger causal for the other in a 2-state MS-VAR model. The conditions given here are specified for a 4-state MS-VAR model where all possible causality classifications are allowed. In a 2-state MS-VAR model one can only test for Granger causality by allowing two regimes and discarding the possibility of other types of causal relationships. The cases given in Warne (2000) correspond to $H_{0}^{2}, H_{0}^{3}, H_{0}^{6}$, and $H_{0}^{7}$ given in Table 1. Compared to Warne's model our specification allows for a more complete regime inference. That is, inferred probabilities can be computed for each of the four regimes and full sample causality tests can be performed.

${ }^{10}$ If the error term $\varepsilon_{t}$ in equation (8) is conditionally normal and that the matrix $P$ has either full rank or equal to one, Warne (2000) shows that the Granger causality in mean-variance implies Granger causality in distribution.
} 
no information in $\mathfrak{R}_{t}$ for predicting $S_{2, t+1}$. This case corresponds to the noncausality of inflation for inflation uncertainty and given as the hypothesis $H_{0}^{5}$ in Table 1.

It is also possible to consider two subcases of the Granger noncausality conditions given in equations (14) and (15) and impose restrictions accordingly that can be tested. The first case arises when $\pi_{t}$ follows a single-regime while $h_{t}$ follows a two-regime process $\left(q_{1}=1\right.$ and $\left.q_{2}=2\right)$. In this case, $h_{t}$ may be noncausal for $\pi_{t}$, if it fails to have an effect through the second channel. That is, $h_{t}$ is noncausal for $\pi_{t}$, if it fails to improve the one-step-ahead prediction of $\pi_{t}$. Similarly, $\pi_{t}$ is noncausal for $h_{t}$, if it fails to improve the one-step-ahead prediction of $h_{t}$. This type of conditional Granger noncausality is specified under the null hypothesis of $H_{0}^{2}$ for noncausality of inflation uncertainty and $H_{0}^{6}$ for noncausality of inflation. The second case occurs when $\pi_{t}$ follows a two-regime while $h_{t}$ follows a single-regime process $\left(q_{1}=2\right.$ and $\left.q_{2}=1\right)$. Under this case, $\pi_{t}$ does not directly depend on the regime in addition to failure of the $h_{t}$ to improve the one-step-ahead prediction of $\pi_{t}$. This is given as the hypothesis $H_{0}^{3}$ for the noncausality of inflation uncertainty for inflation and $H_{0}^{7}$ for the noncausality of inflation for inflation uncertainty.

Finally, inflation uncertainty $h_{t}$ can be noncausal for inflation $\pi_{t}$ when it is conditionally uninformative about the regime. Under conditions of the hypothesis $H_{0}^{4}$ given in Table $1, h_{t}$ is conditionally uninformative about the regime and noncausal for $\pi_{t}$. Analogously, $H_{0}^{8}$ gives the conditions for $\pi_{t}$ for being conditionally uninformative about the regime and noncausal for $h_{t}$. We note that the states in this model arise by imposing the relevant conditions in Table 1 and these are restrictions for testing a specific Granger noncausality hypothesis. 
Table 1: Noncausality and conditional regime independence restrictions in 2-state and 4-state MS-VAR $(p)$ models with $X_{t}=\left[\pi_{t}, h_{t}\right]^{\prime}$

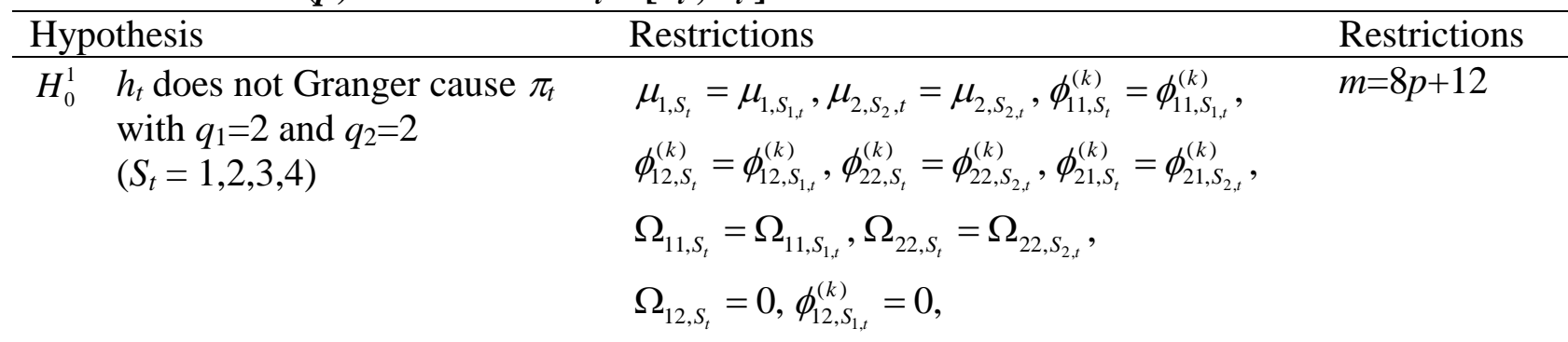

$H_{0}^{2} \quad h_{t}$ does not Granger cause $\pi_{t}$ with $q_{1}=1$ and $q_{2}=2\left(S_{t}=1,3\right)$

$$
\begin{aligned}
& { }_{1, S_{t}}={ }_{1}, \quad{ }_{11, S_{t}}^{(k)}={ }_{11}^{(k)}, \quad{ }_{12, S_{t}}^{(k)}=0, \quad m=3 p+4 \\
& { }_{11, S_{t}}={ }_{11}, \quad{ }_{12, S_{t}}=0 \\
& { }_{2, S_{t}}={ }_{2}, \quad{ }_{21, S_{t}}^{(k)}={ }_{21}^{(k)}, \quad{ }_{22, S_{t}}^{(k)}={ }_{22}^{(k)}, \quad m=4 p+4 \\
& { }_{22, S_{t}}={ }_{22}, \quad{ }_{12, S_{t}}=0, \quad \stackrel{(k)}{12, S_{t}}=0 \text {, }
\end{aligned}
$$

$H_{0}^{3} \quad h_{t}$ does not Granger cause $\pi_{t}$ with $q_{1}=2$ and $q_{2}=1\left(S_{t}=1,2\right)$

$H_{0}^{4} \quad h_{t}$ does not Granger cause $\pi_{t} \quad p_{11}^{(1)}=p_{21}^{(1)}, \quad p_{11}^{(2)}=p_{21}^{(2)}, \phi_{12, S_{t}}^{(k)}=\phi_{12, S_{1, t}}^{(k)}=0 \quad m=2 p+2$ with $q_{1}=2$ and $q_{2}=2$ $\left(S_{t}=1,2,3,4\right)$ with serially uncorrelated Markov process

$H_{0}^{5} \quad \pi_{t}$ does not Granger cause $h_{t}$ with $q_{1}=2$ and $q_{2}=2$ $\left(S_{t}=1,2,3,4\right)$

$$
\begin{aligned}
& \mu_{1, S_{t}}=\mu_{1, S_{1, t}}, \mu_{2, S_{2}, t}=\mu_{2, S_{2, t}}, \phi_{11, S_{t}}^{(k)}=\phi_{11, S_{1, t}}^{(k)}, \\
& \phi_{12, S_{t}}^{(k)}=\phi_{12, S_{1, t}}^{(k)}, \phi_{22, S_{t}}^{(k)}=\phi_{22, S_{2, t}}^{(k)}, \phi_{21, S_{t}}^{(k)}=\phi_{21, S_{2, t}}^{(k)}, \\
& \Omega_{11, S_{t}}=\Omega_{11, S_{1, t}}, \Omega_{22, S_{t}}=\Omega_{22, S_{2, t},} \\
& \Omega_{21, S_{t}}=0, \phi_{21, S_{2, t}}^{(k)}=0,
\end{aligned}
$$

$H_{0}^{6} \quad \pi_{t}$ does not Granger cause $h_{t}$ with $q_{1}=1$ and $q_{2}=2$ $\left(S_{t}=1,3\right)$

$$
\begin{aligned}
& \mu_{1, S_{t}}=\mu_{1}, \phi_{11, S_{t}}^{(k)}=\phi_{11}^{(k)}, \phi_{12, S_{t}}^{(k)}=\phi_{12}^{(k)} \quad m=3 p+4 \\
& \Omega_{11, S_{t}}=\Omega_{11}, \Omega_{21, S_{t}}=0, \phi_{21, S_{t}}^{(k)}=0
\end{aligned}
$$

$H_{0}^{7} \quad \pi_{t}$ does not Granger cause $h_{t}$

$\mu_{2, S_{t}}=\mu_{2}, \phi_{22, S_{t}}^{(k)}=\phi_{22}^{(k)}, \phi_{21, S_{t}}^{(k)}=0$, with $q_{1}=2$ and $q_{2}=1$ $\left(S_{t}=1,2\right)$

$\Omega_{22, S_{t}}=\Omega_{22}, \Omega_{21, S_{t}}=0$

$H_{0}^{8} \quad \pi_{t}$ does not Granger cause $h_{t}$

$$
p_{21}^{(1)}=p_{22}^{(1)}, \quad p_{21}^{(2)}=p_{22}^{(2)}, \quad \phi_{21, S_{t}}^{(k)}=\phi_{21, S_{2, t}}^{(k)}=0
$$
$m=2 p+2$ with $q_{1}=2$ and $q_{2}=2\left(S_{t}=\right.$ $1,2,3,4)$ with serially uncorrelated Markov process

\footnotetext{
Note: In this table, $\pi_{t}$ denotes the inflation rate, $h_{t}$ denotes the inflation uncertainty estimated using the model defined in equations (1)-(3) and (4) $S_{t}$ is the latent state variable defined in equation (10), $m$ is the number of restrictions, $q_{1}$ and $q_{2}$ indicate the number of regimes for the inflation and inflation uncertainty equations, respectively, other symbols are defined in the text relating to equations (8), (9), and (11).
} 


\section{Empirical Analysis}

\section{Data}

The data consists of monthly unadjusted consumer price index based inflation rates from 1921:01 to 2012:12 with a total of 1104 observations. These data are obtained from the Global Financial Database. Inflation rates are constructed by taking 100 times the first difference of the natural $\log$ of the consumer price index. The first 25 observations are lost after the logarithmic transformation and the computation of the transition variables, reducing the total number to 1079 observations.

\section{Nonlinearity test}

We start the empirical analysis by selecting the autoregressive order for a seasonal linear ARFI model of the inflation variables. This model is estimated for different autoregressive orders $p$, where the maximum order is fixed at $p_{\max }=12$. Based on the BIC criterion, the appropriate order is $p=5$. The next step consists of testing linearity against seasonal FISTAR, based on $\mathrm{LM}_{k}$-type tests of nonlinearity, where $k=3$ is the Taylor approximation order. Linearity is tested against the three specifications of the SEA-FISTAR model discussed in section 3.1. Transition variables should be free from seasonality (see Franses et al., 2000). Thus we use as potential transition variables the seasonal difference of $y_{t}$, that is, $\Delta_{12} y_{t-l}=y_{t-l}-y_{t-l-12}$ with $l=1, \cdots, 12$. We found that, for each of the three SEA-FISTAR model specifications, the null hypothesis of linearity is rejected at the 5\% significance level under various transition variables. We select the transition variable that corresponds to the minimal $p$-value. The selected transition variables are $\Delta_{12} y_{t-7}$ for model 1 and $\Delta_{12} y_{t-6}$ for model 2. For model 3, we select $\Delta_{12} y_{t-6}$ and $\Delta_{12} y_{t-9}$ as transition variables in the functions $F($.$) and G($.$) respectively.$

\section{Estimation Results of the SEA-FISTAR-APGARCH Model}

After selecting the transitions variables using $L M$-type tests, the next step is the estimation of different specifications of the SEA-FISTAR-APGARCH model. We use the approximate maximum likelihood method discussed previously. The estimation results of the three specifications of the SEA-FISTAR-APGARCH model are reported in Table 2. Recall, we estimate the SEA-FISTAR-APGARCH model recursively, however, in Table 2, we report the full-sample estimates. For the SEA-FISTAR part, the estimated differencing parameter $\hat{d}$ ranges from 0.449 to 0.483 and is significant at the $5 \%$ level. This suggests strong evidence of long memory in inflation rates. The estimated threshold parameter $\hat{c}$ is equal to 0.5488 and -1.0215 in model 1 and model 2, respectively. For model 3, the threshold parameters $\hat{c}_{G}$ and $\hat{c}_{F}$ are equal to 0.0999 and -1.0215 , respectively. All threshold parameters are significant at the 5\% level. Comparing $\hat{\phi}_{1, i}$ with $\hat{\phi}_{2, i}$ we can observe that in all cases there are different regimes in the autoregressive parameters. The estimates of the seasonal means $\left(\hat{\mu}_{1, s}\right.$ and $\left.\hat{\mu}_{2, s}, s=1, \cdots, 12\right)$ are not reported here for space considerations but their results indicate the existence of seasonal behaviour in the inflation rates.

Residual serial correlation tests based on $L M$ statistics provide strong evidence for no residual serial correlation for all seasonal FISTAR model specifications. The large values of the estimates of threshold parameters $\widehat{\gamma}_{G}$ and $\hat{\gamma}_{F}$ indicate that transition from one regime to another occurs suddenly at the estimated threshold parameters. For the APGARCH part, the ARCH and GARCH terms of the variance equation are highly significant and having similar values for all three specification of the model. 
Table 2: Summary of estimated models for South African inflation rates

\begin{tabular}{|c|c|c|c|}
\hline & Model 1 & Model 2 & Model 3 \\
\hline \multicolumn{4}{|l|}{ SEA-FISTAR part } \\
\hline$\hat{d}$ & $0.4835^{* * *}$ & $0.4600^{* * *}$ & $0.4489^{* * * *}$ \\
\hline$\hat{c}_{G}$ & $0.5488^{* * * *}$ & $-1.0215^{* * * *}$ & $0.0999^{* * *}$ \\
\hline$\hat{\gamma}_{G}$ & 258.2647 & 404.2926 & 44.834 \\
\hline$\hat{c}_{F}$ & & & $-1.0215^{* * *}$ \\
\hline$\hat{\gamma}_{F}$ & & & 686.6245 \\
\hline$\hat{\phi}_{1,1}$ & $-0.3219^{* * * *}$ & $-0.4638^{* * * *}$ & $-0.2352^{* * *}$ \\
\hline$\hat{\phi}_{1,2}$ & $-0.2204^{* * * *}$ & $-0.4412^{* * * *}$ & $-0.2533^{* * *}$ \\
\hline$\hat{\phi}_{1,3}$ & $-0.1418^{* * *}$ & -0.2007 & $-0.1879^{* * *}$ \\
\hline$\hat{\phi}_{1,4}$ & $-0.1703^{* * *}$ & $-0.2689^{* * *}$ & -0.0373 \\
\hline$\hat{\phi}_{1,5}$ & $-0.1761^{* * *}$ & -0.1065 & -0.0583 \\
\hline$\hat{\phi}_{2,1}$ & $-0.3099^{* * *}$ & $-0.2792^{* * *}$ & $-0.3525^{* * *}$ \\
\hline$\hat{\phi}_{2,2}$ & $-0.3366^{* * *}$ & $-0.1987^{* * * *}$ & $-0.1421^{* *}$ \\
\hline$\hat{\phi}_{2,3}$ & $-0.2984^{* * * *}$ & $-0.1482^{* * * *}$ & $-0.0962^{*}$ \\
\hline$\hat{\phi}_{2,4}$ & 0.0256 & $-0.1144^{* * *}$ & $-0.2186^{* * *}$ \\
\hline$\hat{\phi}_{2,5}$ & 0.0368 & $-0.1109^{* * *}$ & $-0.1605^{* * *}$ \\
\hline $\mathrm{SC}(1)$ & $0.892(0.345)$ & $2.283(0.131)$ & $1.819(0.178)$ \\
\hline $\mathrm{SC}(4)$ & $1.428(0.222)$ & $0.744(0.562)$ & $1.747(0.137)$ \\
\hline \multicolumn{4}{|l|}{ APGARCH part } \\
\hline$\omega$ & $0.0508^{* * * *}$ & $0.0529^{* * *}$ & $0.0625^{* * *}$ \\
\hline$\alpha$ & $0.1779^{* *}$ & $0.1547^{* * *}$ & $0.1446^{* * * *}$ \\
\hline$\beta$ & $0.7426^{* * * *}$ & $0.7733^{* * *}$ & $0.7659^{* * *}$ \\
\hline$\delta$ & 0.2736 & 0.1968 & 0.2323 \\
\hline$\theta$ & 0.2003 & 0.0688 & 0.1240 \\
\hline
\end{tabular}

Note: ${ }^{* * * *},{ }^{* *}$ and ${ }^{*}$ represent significance at the $1 \%, 5 \%$, and $10 \%$ levels, respectively. $p$-values are given in parentheses.SC $(q)$ denotes the $F$ variant of LM test of no serial correlation in residuals up to order $q$.Model 1 is a SEA-FISTAR-APGARCH model, which allows only non-seasonal parameters to switch across regimes. Model 2 is a SEA-FISTAR-APGARCH model, which allows both seasonal and non-seasonal parameters to switch simultaneously across regimes with a single switching function. Model 3 is a SEA-FISTAR-APGARCH model, which allows both seasonal and non-seasonal parameters to switch under different regimes.

The inflation uncertainty is estimated as the conditional variance appearing in the SEA-FISTAR model in (1)-(3), which is specified to follow the APGARCH(1,1) model in (4).The South African monthly inflation rate and the estimated inflation uncertainties from the three SEA-FISTAR-APGARCH specifications are presented in Figure 1. As expected, the inflation rate appears to be less volatile than the inflation uncertainties. The impact of the depression during the 1930s is evident from the high levels of inflation associated with high levels of inflation uncertainty. Following the oil price shocks of the 1970s inflation continue to rise, reaching its highest level in the mid-1980s. The period of inflation targeting, commencing in 1990, brought about consistently lower levels of inflation. The descriptive statistics of these series are presented in Table 3.The mean value for the inflation variable is 0.46 and it is about $50 \%$ larger than the mean 
inflation uncertainty from the different models which are about 0.24 . The skewness is relatively greater than zero in all cases showing the series are somewhat skewed and the kurtosis deviates from 3 indicating they series are in general not normally distributed. This is confirmed by the Jarque-Bera normality test which is rejected at the $1 \%$ significance level for all series. Also, the Ljung-Box Q first and fourth autocorrelation tests are rejected for all the series. This means that both inflation and inflation uncertainty series have autocorrelation of the first and fourth order. The Lagrange multiplier (LM) tests for autoregressive condition heteroskedasticity (ARCH) is rejected at $1 \%$ significance level for all series implying that all the series exhibit significant ARCH effects.

Figure 1: Inflation and inflation uncertainty series

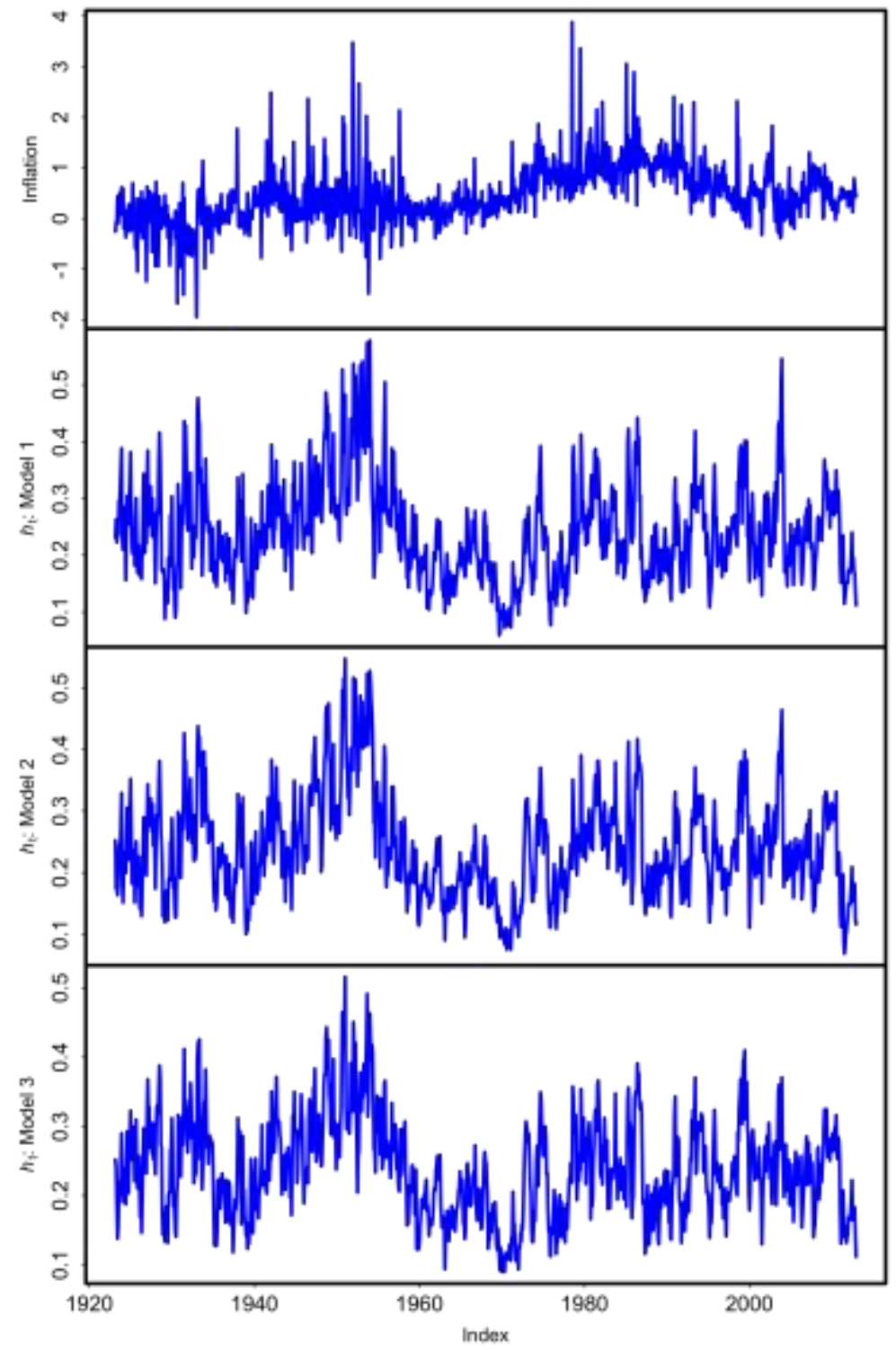

Note: Figure plots the inflation and inflation uncertainty series. The sample period covers March 1923-Decmber 2012 with $n=1078$ observations. Inflation is the monthly inflation rate. Inflation uncertainty is obtained from three model specifications defined in equations (1)-(4). Model 1 is a SEA-FISTAR-APGARCH model, which allows only non-seasonal parameters to switch across regimes. Model 2 is a SEA-FISTAR-APGARCH model, which allows both seasonal and non-seasonal parameters to switch simultaneously across regimes with a single switching function. Model 3 is a SEA-FISTAR-APGARCH model, which allows both seasonal and non-seasonal parameters to switch under different regimes. 
Table 3: Descriptive statistics

\begin{tabular}{lrrrr}
\hline & \multicolumn{2}{c}{$\begin{array}{c}\text { Inflation } \\
\text { uncertainty from uncertainty from }\end{array}$} & \multicolumn{2}{c}{ uncertainty from } \\
& Inflation & Model 1 & Model 2 & Model 3 \\
\hline Mean & 0.4612 & 0.2434 & 0.2422 & 0.2394 \\
S.D. & 0.5833 & 0.086 & 0.0829 & 0.0712 \\
Min & -1.9443 & 0.0593 & 0.0684 & 0.09 \\
Max & 3.8772 & 0.578 & 0.5476 & 0.5155 \\
Skewness & 0.7701 & 0.789 & 0.7255 & 0.4837 \\
Kurtosis & 3.4561 & 0.8237 & 0.5822 & 0.1511 \\
JB & $647.0830^{* * *}$ & $143.1610^{* * *}$ & $110.4010^{* * * *}$ & $43.2590^{* * * *}$ \\
$Q(1)$ & $255.0076^{* * *}$ & $766.1288^{* * *}$ & $836.5604^{* * *}$ & $784.0646^{* * * *}$ \\
$Q(4)$ & $255.0076^{* * *}$ & $766.1288^{* * *}$ & $836.5604^{* * * *}$ & $784.0646^{* * * *}$ \\
ARCH(1) & $8.1893^{* * * *}$ & $503.7468^{* * *}$ & $674.9368^{* * *}$ & $500.1503^{* * * *}$ \\
ARCH(4) & $14.6041^{* * *}$ & $512.7470^{* * *}$ & $677.2952^{* * *}$ & $506.2271^{* * * *}$ \\
$n$ & 1078 & 1078 & 1078 & 1078 \\
\hline
\end{tabular}

Note: This table reports the descriptive statistics for inflation and inflation uncertainty series. The sample period covers March 1923 to December 2012 with $n=1078$ observations. Inflation is the monthly inflation rate. Inflation uncertainty is obtained from three model specifications defined in equations (1)-(4). Model 1 is a SEA-FISTAR-APGARCH model, which allows only non-seasonal parameters to switch across regimes. Model 2 is a SEA-FISTAR-APGARCH model, which allows both seasonal and non-seasonal parameters to switch simultaneously across regimes with a single switching function. Model 3 is a SEA-FISTAR-APGARCH model, which allows both seasonal and non-seasonal parameters to switch under different regimes. In addition to the mean, the table reports standard deviation (S.D.), minimum (min), maximum (max), skewness and kurtosis statistics, the Jarque-Bera normality test (JB), the Ljung-Box Q first $[Q(1)]$ and the fourth $[Q(4]$ order autocorrelation tests, and the first $[\mathrm{ARCH}(1)]$ and the fourth $[\mathrm{ARCH}(4)]$ order Lagrange multiplier (LM) tests for the autoregressive conditional heteroskedasticity (ARCH). ${ }^{* * *},{ }^{* *}$ and ${ }^{*}$ represent significance at the $1 \%, 5 \%$, and $10 \%$ levels, respectively.

\section{Granger Causality Test Results}

Prior to testing for asymmetric and time-varying causality between inflation and inflation uncertainty, we first present the results from the standard linear Granger causality test under the VAR model for each inflation uncertainty in the lower panel of Table 4.The order of the VAR and MS-VAR model is selected by the BIC. The BIC uniquely selects an order of 4 in each case. The hypothesis that inflation does not Granger cause inflation uncertainty cannot be rejected for models 2 and 3 but is weakly rejected for model 1 . In all cases the hypothesis that inflation uncertainty does not Granger cause inflation cannot be rejected. Overall, the linear model does not appear to support either the Friedman nor the Cukierman-Meltzer hypotheses. However, the validity of these results depends on the validity of the linearity assumption in the standard Granger causality test. Therefore we present a series of model selection criteria (the log likelihood, Akaike information criterion and LR statistics) for linear bivariate VAR and MS-VAR with four states in the upper panel of Table 4. All three tests select the MS-VAR as the best model. Particularly, the LR statistics which tests the linear VAR model under the null hypothesis against the MS-VAR with 4-states under the alternative hypothesis is rejected at the $1 \%$ significance level based on both the $\chi^{2} p$-values and $p$-values of the Davies (1987) test. 
Table 4: Model selection criteria, linearity, and linear Granger causality tests

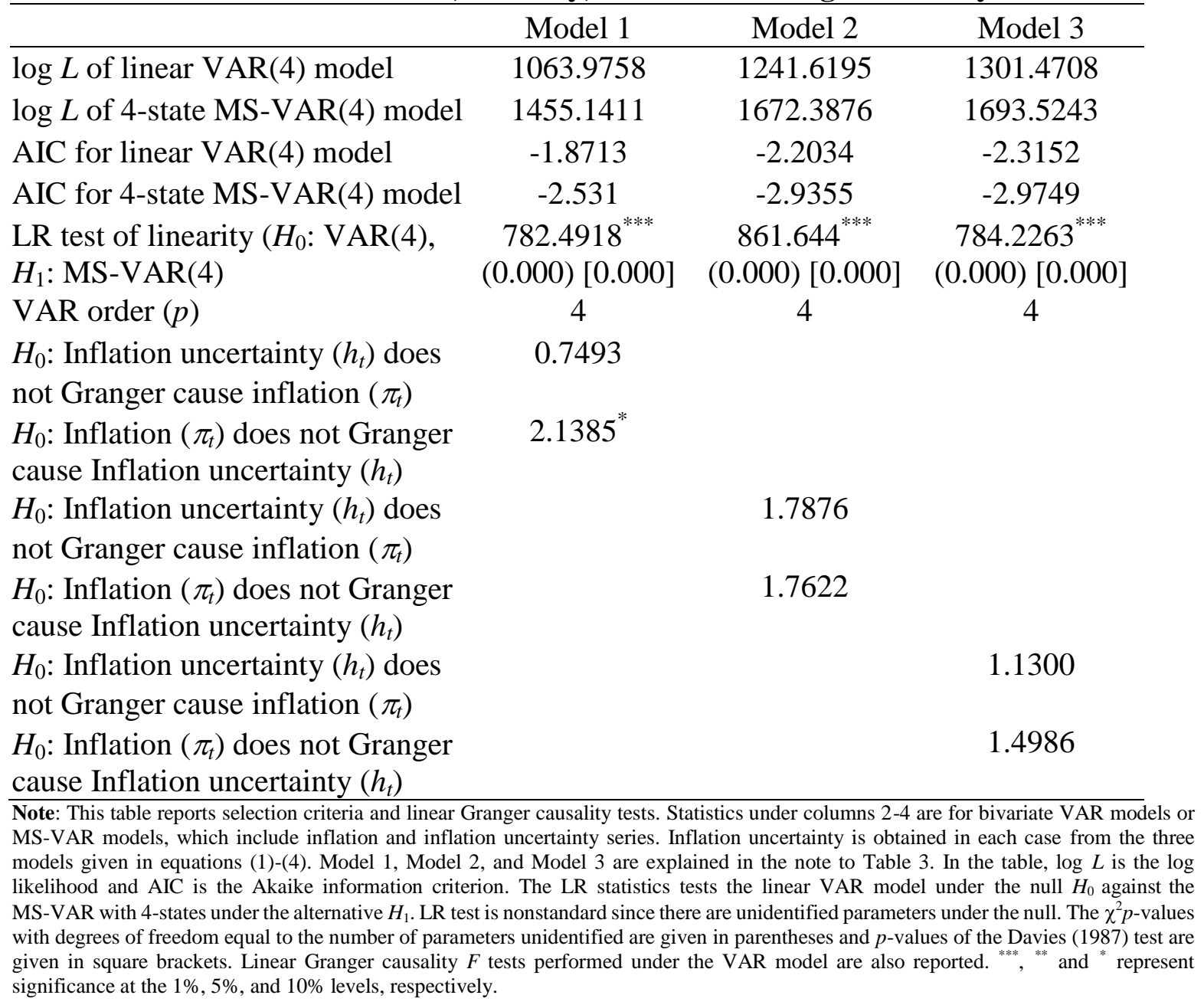

Having established the adequacy of the MS-VAR(4) model, the subsequent discussion presents the results for the asymmetric and time-varying causality using inflation uncertainty from Model 3 and the inflation rate. Results for Models 1 and 2 are analogues and available from the authors upon request. The parameters of the Markov switching model is estimated using the expectation maximization (EM) algorithm (Lindgren 1978; Hamilton 1990, 1994) assuming that the conditional distribution of $X_{t}$ given $\left\{{ }_{t}, S_{t}, S_{t 1}, \ldots, S_{0} ;\right\}$ is normal. The likelihood function is numerically approximated using the EM algorithm and the ML estimates are found by the Broyden-Fletcher-Goldfarb-Shanno (BFGS) optimization algorithm. The asymptotic standard errors of estimates are computed using the Sandwich estimator. Table 5 reports the transition probabilities, the ergodic probabilities, the expected duration of system in each regime, and the direction of the causal relationship between inflation and its uncertainty in the four-regime MS-VAR model. The causal links between inflation and its uncertainty are different in the four regimes. In regime 4, the causal link between inflation and its uncertainty is bi-directional. Inflation uncertainty has predictive power for inflation in regime 2, while inflation has predictive power for its uncertainty in regime 3 . There is no causal link between the series in regime 1 .

The expected duration of the system in regimes 1, 2, 3, and 4 are 13.57, 2.31, 5.59 and 1.06 months, respectively. These indicate that the expected duration of bi-directional causal links 
between the series considered is about 1.06 months. Also, the expected duration of causal link from inflation to its uncertainty is about 2.31 months, while the duration for the link from inflation uncertainty to inflation is about 5.59 months. According to the results of the expected duration of system in regimes 2 and 3, inflation seems to have a very strong predictive content for inflation uncertainty compared to causality from inflation uncertainty to inflation. The results indicate that the impact of inflation on its uncertainty is positive in regime 4 and the effect of inflation uncertainty on inflation is also positive in regime 4. The effect of inflation on its uncertainty is positive in regime 3 , and the effect of inflation uncertainty on inflation is positive in regime 2. We also compute the weighted coefficients for the sign of the effect. Since the total impact will depend on the combination of regimes 2 and 4 for the effect of inflation uncertainty on inflation and the combination of regimes 3 and 4 for the effect of inflation on inflation uncertainty, we calculate the weighted sums of the impacts in different regimes. These weighted impacts are calculated by weighting the corresponding regime coefficient sums with the normalized ergodic probabilities. The results indicate that the sign of the total weighted effect between the series is positive for lead-lag relationship in both directions. The regime inference reported in Table 5 shows that the number of observation is zero for inflation uncertainty Granger causing inflation and is 313 for inflation Granger causing inflation uncertainty.

Table 5: Estimates of the 4-state MS-VAR model

\begin{tabular}{lcccc} 
& \multicolumn{3}{l}{} \\
\cline { 2 - 5 } & $p_{1 j}$ & $p_{2 j}$ & $p_{3 j}$ & 0.480 \\
Regime 1 & 0.926 & 0.428 & 0.179 & 0.000 \\
Regime 2 & 0.000 & 0.567 & 0.000 & 0.465 \\
Regime 3 & 0.073 & 0.000 & 0.821 & 0.054
\end{tabular}

Regime 1

Regime 2

Regime 3

Regime 4

Regime 1

Regime 2

Regime 3

Regime 4

Weighted Coefficients
Ergodic

Probabilities

$\begin{array}{lcc}0.709 & 13.569 & 761 \\ 0.000 & 2.307 & 0 \\ 0.291 & 5.586 & 313 \\ 0.000 & 1.057 & 0\end{array}$

Effect of Inflation Uncertainty on Inflation

Effect of Inflation on Inflation Uncertainty

\begin{tabular}{cc}
\hline-- & -- \\
0.440 & -- \\
-- & 0.002 \\
1.160 & 0.032 \\
0.836 & 0.002
\end{tabular}

Ergodic Probability

Number of Observations

$\frac{\text { Regime Inference }}{\text { Inflation Uncert }}$

\begin{tabular}{cc}
\hline $\begin{array}{c}\text { Inflation Uncertainty Granger Causes } \\
\text { Inflation }\end{array}$ & $\begin{array}{c}\text { Inflation Granger Causes Inflation } \\
\text { Uncertainty }\end{array}$ \\
\hline 0.000 & 0.291 \\
0 & 313
\end{tabular}

Note: This table reports the estimates of the transition probabilities, the ergodic probabilities, the expected duration of system in each regime, and the impact between inflation and inflation uncertainty in the four-regime MS-VAR model. $p_{i j}, i, j=1,2,3,4$, denote the transition probabilities defined in equation (9). $n_{i}$ denote the number of observation falling in regime $i$ estimated from the limiting ergodic probabilities. The weighted coefficients for the impacts of inflation uncertainty on inflation and inflation on inflation uncertainty are calculated by weighting the corresponding regime coefficient sums with the normalized ergodic probabilities. 
Further insight about the changes in causality throughout the sample period may be obtained by looking at the inferred probabilities for the Granger causality regime between inflation and inflation uncertainty from the MS-VAR model in Figure 2. The plots shown in Panel A of Figure 2, is the inferred probability of inflation Granger causing inflation uncertainty, which are the sum of the filtered probabilities of regime 3 and regime 4 . The plots given in Panel B of Figure 2 is the inferred probability of inflation uncertainty Granger causing inflation, which are the sum of the filtered probabilities of regime 2 and regime 4. The plot of the inferred probabilities shown in Panel A of Figure 1 indicates that inflation has positive predictive content for inflation uncertainty for most of sample period. This finding suggests strong evidence for the Friedman hypothesis during the subsamples of the study period. The probability estimates for Panel B are essentially almost all zero. This indicates that inflation uncertainty has no predictive content for inflation.

\section{Figure 2: Estimates of the inferred filtered probabilities for the Granger Causality}
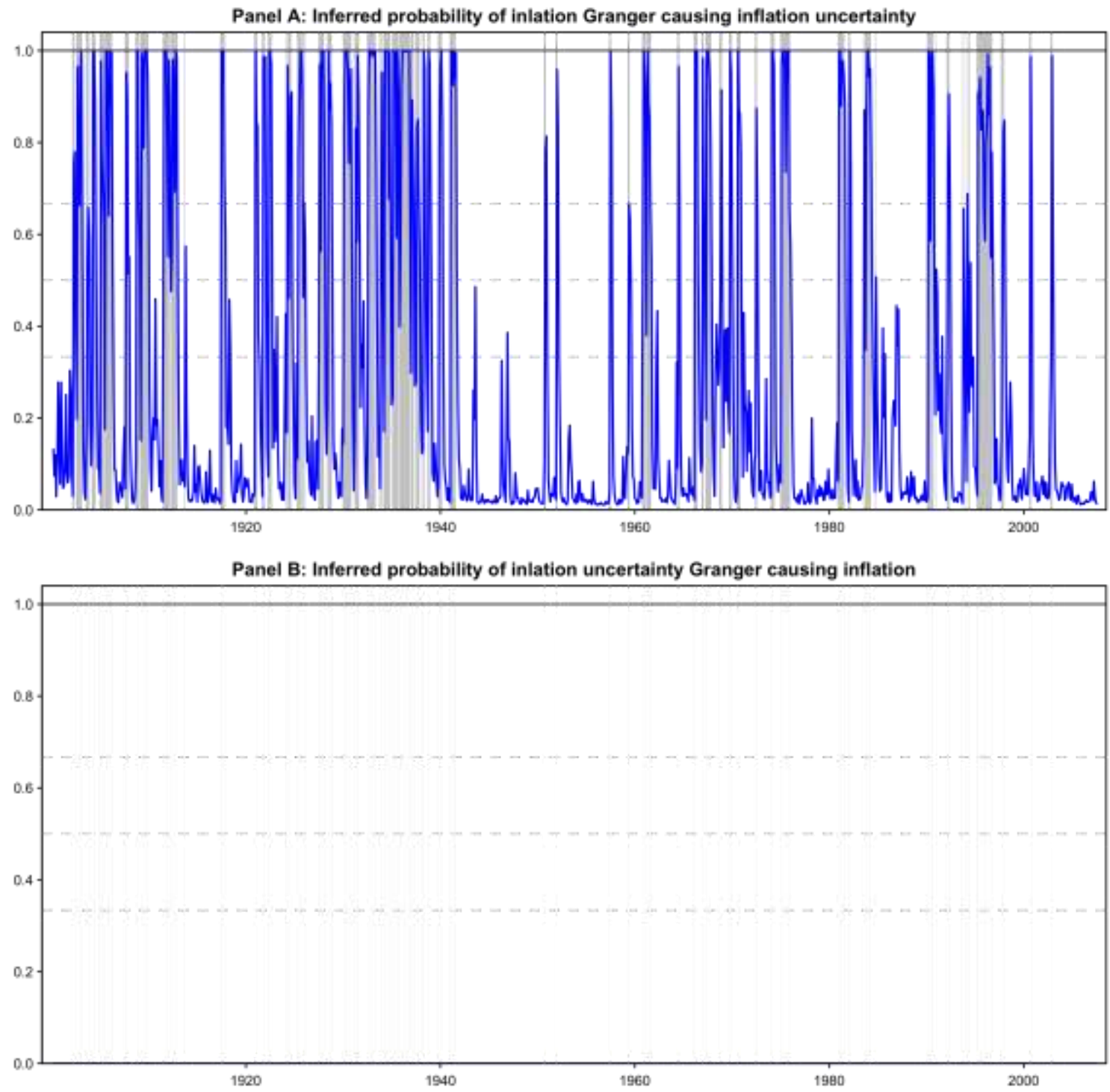

Notes: Panels A plots the inferred probability of inflation Granger causing inflation uncertainty, which is the sum of the regime 3 and regime 4 filtered probabilities. Similarly, Panel B reports the filtered probability of inflation uncertainty Granger causing inflation, which is the sum of the regime 2 and regime 4 filtered probabilities. The regions corresponding to maximum probability are shaded. 
We test for conditional Granger causality between inflation and inflation uncertainty using the four sufficient conditions. The $F$ statistics and corresponding $p$-values from testing the hypothesis that $h_{t} \neq>\pi_{t}\left(\alpha_{1}^{(k)}=0\right.$ and $\left.\phi_{12, S_{t}}^{(k)}=0, S_{t}=\{1,2,3,4\}\right)$ and that $\pi_{t} \neq>h_{t}\left(\alpha_{2}^{(k)}=0\right.$ and $\left.\phi_{21, S_{t}}^{(k)}=0, S_{t}=\{1,2,3,4\}\right)$ are reported in Table 6 . For testing that $h_{t} \neq>\pi_{t}$, the evidence obtained from the $F$ statistics, shows that the hypothesis that the coefficients on the lagged inflation uncertainty are zero in the inflation equation cannot be rejected. Hence, we find no evidence of conditional (i.e., lead-lag relationship) Granger causality from inflation uncertainty to inflation. The same holds when either of the variables is restricted to follow a single known regime. Conversely, for testing that $\pi_{t} \neq>h_{t}$, the evidence obtained from the $F$ statistics shows that the hypothesis that the coefficients on the lagged inflation are zero in the inflation uncertainty equation is rejected at the $1 \%$ significance level. Thus, there is strong evidence of conditional (i.e., lead-lag relationship) Granger causality from inflation to inflation uncertainty. The same holds when either of the variables is restricted to follow a single known regime as well as whether the regimes are identified by causality direction or unidentified. These findings corroborate the evidence obtained from the inferred probabilities given in Figure 2.

\section{Table 6: Conditional Granger noncausality in 2-state and 4-state MS-VAR(4) model of inflation and inflation uncertainty}

\begin{tabular}{lcccc}
\hline Hypothesis & $m$ & $S$ & $F^{a}$ & $p$ value of $F$ \\
\hline$q_{1}=2, q_{2}=2$ & & & & \\
$H_{0}:{ }_{1}^{(k)}=0$ & 4 & 38 & 0.7432 & 0.5626 \\
$H_{0}:{ }_{2}^{(k)}=0$ & 4 & 38 & $5.6702^{* * *}$ & 0.0002 \\
$q_{1}=1, q_{2}=2$ & & & & \\
$H_{0}:{ }_{1}^{(k)}=0$ & 4 & 14 & 0.9080 & 0.4585 \\
$H_{0}:{ }_{2}^{(k)}=0$ & 4 & 12 & $6.9292^{* * *}$ & $<0.0001$ \\
$q_{1}=2, q_{2}=1$ & & & & \\
$H_{0}:{ }_{1}^{(k)}=0$ & 4 & 12 & 0.8584 & 0.4884 \\
$H_{0}:{ }_{2}^{(k)}=0$ & 4 & 14 & $5.5428^{* * *}$ & 0.0002 \\
$q_{1}=2, q_{2}=2$ & & & & \\
$H_{0}:{ }_{12, S_{t}}=0^{\mathrm{b}}$ & 16 & 40 & 1.2450 & 0.2264 \\
$H_{0}:{ }_{21, S_{t}}^{(k)}=0^{\mathrm{c}}$ & 16 & 40 & $3.1437^{* * *}$ & $<0.0001$ \\
\hline
\end{tabular}

\footnotetext{
Note: $m$ denotes the number restrictions. ${ }^{* * *},{ }^{* *}$ and ${ }^{*}$ represent significance at the $1 \%, 5 \%$, and $10 \%$ levels, respectively. $<$ designates less than the number it precedes.

${ }^{\text {a }}$ For the $F$ statistic the reference distribution is $F(m, n-s)$ and the $F$ statistic is computed from $F=((n-s) /(m n)) W$, where $W$ is the Wald statistic, $n$ is the number of observations, $s$ is the closest integer to the average number of free parameters per equation under $H_{0}$, i.e., $s=\operatorname{int}[(k-m) / 2]$, where $k$ is the total number of parameters estimated.

${ }^{b}$ Test of the null hypothesis that inflation uncertainty does not Granger cause inflation in an unrestricted MS-VAR(4) model with 4 states.

${ }^{\mathrm{c}}$ Test of the null hypothesis that inflation does not Granger cause inflation uncertainty in an unrestricted MS-VAR(4) model with 4 states.
}

It is observed that the filtered probabilities (Panel A of Figure 2) indicate occasional shifts in the causal links between inflation and its uncertainty. The inferred probability estimates presents strong evidence of time varying causal relationships between the two series. Therefore, there is a 
need to test whether the four types of causal relationships hold for the whole sample. This can be done using the four types of restrictions given in Table 1 and corresponding Wald tests. In the first case, we test the null hypothesis that the inflation uncertainty does not Granger cause inflation, $H_{0}^{1}$ , and both inflation and inflation uncertainty are allowed to follow two independent states. The second hypothesis is that the inflation uncertainty does not Granger cause inflation, $H_{0}^{2}$, when inflation follows a fixed single regime and inflation uncertainty is free to follow two regimes. In the third case, we test the hypothesis that inflation uncertainty does not Granger cause inflation, $H_{0}^{3}$, when inflation uncertainty follows a single regime while inflation has two regimes. In the fourth case, the hypothesis $H_{0}^{4}$, inflation uncertainty is conditionally uninformative about the regime and noncausal for inflation. When we wish to test the hypothesis that inflation uncertainty is Granger noncausal in mean (and variance) for inflation, the restrictions given under the hypotheses $H_{0}^{1}, H_{0}^{2}$, and $H_{0}^{3}$ imply that inflation uncertainty is conditionally uninformative about the regime, while $H_{0}^{4}$ implies that inflation does not directly depend on the regime (other than the dependence via residual covariance). Moreover, the $H_{0}^{1}, H_{0}^{2}$, and $H_{0}^{3}$ restrictions allow the Markov process to be serially correlated, while $H_{0}^{4}$ does not. The four cases given in Table 1 are also extended to the case where under the null hypothesis inflation does not Granger cause inflation uncertainty. The corresponding null hypothesis is again tested under the four cases. In the first case, $H_{0}^{5}$, both inflation and inflation uncertainty follow two regimes. In the second case, inflation is restricted to a single fixed regime, $H_{0}^{6}$. The third case, $H_{0}^{7}$, restricts inflation uncertainty to a single fixed regime while inflation is free to follow two regimes. The fourth case, $H_{0}^{8}$, restricts inflation uncertainty not to depend on the regime directly.

The estimation results of the Wald tests for each of the eight hypotheses are given in Table 7. With regard to the hypothesis $h_{t} \neq>\pi_{t}$, the four sets of sufficiency restrictions relating to $H_{0}^{1}$, $H_{0}^{2}, H_{0}^{3}$, and $H_{0}^{4}$ cannot be rejected at any of the conventional significance levels. Therefore, the null hypotheses that inflation uncertainty is conditionally uninformative about the regime $\left(H_{0}^{1}\right.$, $H_{0}^{2}$, and $H_{0}^{3}$ ), and the null hypothesis that inflation does not directly depend on the regime (other than via the residual covariances) cannot be rejected. Overall, the non-rejection of all four hypothesis suggest the non-existence of regime-prediction Granger causality. Conversely, the four sets of sufficiency restrictions relating to $H_{0}^{5}, H_{0}^{6}, H_{0}^{7}$ and $H_{0}^{8}$ are strongly rejected at the $1 \%$ significance level. The overall rejections suggest the existence of regime-prediction Granger causality. Thus, inflation helps to predict the regime of inflation uncertainty. That is, the inflation process contains unique information for predicting the regime process of inflation uncertainty. These findings are robust to the cases where either of the series is restricted to follow a single regime (i.e. whole sample) and the cases where they are allowed to follow a two regime process. 
Table 7: Wald tests of the Granger noncausality restrictions in the 2-state and 4-state MS-VAR(4) model of inflation and inflation uncertainty

\begin{tabular}{|c|c|c|c|c|c|}
\hline \multicolumn{2}{|c|}{ Hypothesis } & $m$ & $s$ & $F^{a}$ & $p$-value of $F$ \\
\hline$H_{0}^{1}$ & $\begin{array}{l}h_{t} \text { does not Granger cause } \pi_{t} \text { with } q_{1}=2 \\
\text { and } q_{2}=2\left(S_{t}=1,2,3,4\right)\end{array}$ & 44 & 26 & 0.9782 & 0.5136 \\
\hline$H_{0}^{2}$ & $\begin{array}{l}h_{t} \text { does not Granger cause } \pi_{t} \text { with } q_{1}=1 \\
\text { and } q_{2}=2\left(S_{t}=1,3\right)\end{array}$ & 16 & 16 & 1.0135 & 0.4391 \\
\hline$H_{0}^{3}$ & $\begin{array}{l}h_{t} \text { does not Granger cause } \pi_{t} \text { with } q_{1}=2 \\
\text { and } q_{2}=1\left(S_{t}=1,2\right)\end{array}$ & 20 & 14 & 1.0326 & 0.4194 \\
\hline$H_{0}^{4}$ & $\begin{array}{l}h_{t} \text { does not Granger cause } \pi_{t} \text { with } q_{1}=2 \\
\text { and } q_{2}=2\left(S_{t}=1,2,3,4\right) \text { with serially } \\
\text { uncorrelated Markov process }\end{array}$ & 10 & 43 & 0.8248 & 0.6047 \\
\hline$H_{0}^{5}$ & $\begin{array}{l}\pi_{t} \text { does not Granger cause } h_{t} \text { with } q_{1}=2 \\
\text { and } q_{2}=2\left(S_{t}=1,2,3,4\right)\end{array}$ & 44 & 26 & $1.8812^{* * *}$ & 0.0005 \\
\hline$H_{0}^{6}$ & $\begin{array}{l}\pi_{t} \text { does not Granger cause } h_{t} \text { with } q_{1}=1 \\
\text { and } q_{2}=2\left(S_{t}=1,3\right)\end{array}$ & 20 & 14 & $2.5533^{* * * *}$ & 0.0002 \\
\hline$H_{0}^{7}$ & $\begin{array}{l}\pi_{t} \text { does not Granger cause } h_{t} \text { with } q_{1}=2 \\
\text { and } q_{2}=1\left(S_{t}=1,2\right)\end{array}$ & 16 & 16 & $2.7220^{* * *}$ & 0.0003 \\
\hline$H_{0}^{8}$ & $\begin{array}{l}\pi_{t} \text { does not Granger cause } h_{t} \text { with } q_{1}=2 \\
\text { and } q_{2}=2\left(S_{t}=1,2,3,4\right) \text { with serially } \\
\text { uncorrelated Markov process }\end{array}$ & 10 & 43 & $3.2863^{* * *}$ & 0.0003 \\
\hline
\end{tabular}

Note: $m$ denotes the number restrictions. ${ }^{* * *},{ }^{* * *}$ and ${ }^{*}$ represent significance at the $1 \%, 5 \%$, and $10 \%$ levels, respectively. < designates less than the number it precedes.

${ }^{\text {a }}$ For the $F$ statistic the reference distribution is $F(m, m-s)$ and the $F$ statistic is computed from $F=((T-s) /(m T)) W$, where $W$ is the Wald statistic, $T$ is the number of observations, $s$ is the closest integer to the average number of free parameters per equation under $\mathrm{H}_{0}$, i.e., $s=$ int $[(k-m) / 2]$, where $k$ is the total number of parameters estimated.

\section{Conclusion}

This paper uses the recursively-estimated SEA-FISTAR-APGARCH type model to estimate inflation uncertainty as the conditional variance of the unanticipated shocks to the South Africa's monthly (seasonally) unadjusted consumer price index based inflation rates covering the period 1921-1 to 2012-12. The SEA-FISTAR-APGARCH type model is flexible and capable of simultaneously dealing with the long memory behaviour, nonlinearity, seasonality and structural breaks in the mean of the inflation series. Further, a recursive, rather than a full-sample, estimation of the SEA-FISTAR-GARCH model allows us to obtain a time-varying measure of uncertainty, which is not only a more realistic depiction of how agents form the uncertainty variable as new data becomes available, but this approach also allows us to tackle for possible structural breaks in the parameter estimates of the APGARCH part of the model, from which the measure of uncertainty is formed. The latter is equally important, since in an emerging country like South Africa, structural changes are not an unlikely event to affect decision making processes. Subsequently, we examine the causal links between inflation and inflation uncertainty. Specifically, the validity of the popular hypotheses by Friedman, Cukierman-Meltzer, Holland, Pourgerani-Maskus and Ungar-Zilberfab about dynamic linkages between inflation and inflation uncertainty are tested. A VAR model with time-varying parameters in which changes in the causality are reflected by time-varying parameters is used. The causality changes are regarded as random events that are controlled by an exogenous Markov switching VAR (MS-VAR) model, where inferences on the causality changes 
are based on the estimated probability that each observation belongs to a specific causality regime. We estimate the MS-VAR model assuming a nonlinear relationship with asymmetry. This procedure provides information on the direction and sign of the Granger causality relationship between inflation and its uncertainty. The estimates of the four-state MS-VAR model show that inflation uncertainty has a positive effect on inflation in regime 2 while inflation has a positive effect on inflation uncertainty in regime 3 . In regime 4, there is a bidirectional causality between inflation and inflation uncertainty while the sign of the effects are zero in regime 1 . One can conclude that inflation and inflation uncertainty are positive determinants of each other depending on which regime is considered. However, evidence based on the inferred probabilities, which is a sum of the filtered probabilities for regimes 2 and 4 for inflation uncertainty Granger causing inflation, and sum of filtered probabilities from regimes 3 and 4 for inflation Granger causing inflation uncertainty shows a strong positive causality from inflation to inflation uncertainty in various subsamples but basically no visible form of causality from the latter to the former. When we implement a conditional Granger non-causality, we observe strong evidence of conditional (i.e. lead-lag relationship) Granger causality from inflation to inflation uncertainty but not vice versa. Further, evidence from the Wald tests shows that inflation has strong regime prediction power for inflation uncertainty irrespective of whether the series are restricted to follow a single regime (i.e. whole sample) or allowed to follow a two regime process. Thus, the inflation process contains unique information for predicting the regime process of inflation uncertainty. However, we could not establish any regime-prediction causality from inflation uncertainty to inflation. Therefore, we conclude evidence in favour of the Friedman's hypothesis while accounting for regime changes.

The results have important policy implications. Since inflation increases inflation uncertainty, the importance of keeping inflation low, stable, and predictable cannot be overemphasized. The monetary authority need to ensure quick, effective and efficient policy response to inflation development in South Africa since this will help to reduce inflation and hence minimize its marginal effect on inflation uncertainty. Moreover, in order to rationalize as well as anchor the public's inflation expectations, information on the various drivers of inflation in South Africa and inflation forecasts needs to be given adequate publicity. These will also assist in communicating the monetary policy stance and hence improve South Africa's Reserve Bank's transparency and accountability. While much is expected from the monetary policy authority, there is need for fiscal policy response as well since both demand and supply shocks may contribute to rising inflation in the economy. Therefore, both monetary and fiscal policies need to be co-ordinated to effectively reduce inflation to a desirable minimum.

\section{References}

Ajmi, A.N., Ben Nasr, A., and Boutahar, M. (2008). Seasonal Nonlinear Long Memory Model in US Inflation Rates. Computational Economics, 31,243-254.

Amusa, K., Gupta, R., Karolia, S. and Simo-Kengne, B.D. (2013). The long-run impact of inflation in South Africa. Journal of Policy Modeling 35, 798-812.

Baillie, R., Chung, C. and Tieslau, M. (1996a). Analyzing inflation by the fractionally integrated ARFIMA-GARCH model. Journal of Applied Econometrics, 11, 23-40.

Baillie, R.T., Bollerslev, T. and Mikkelsen, H.O. (1996b). Fractionally integrated generalized autoregressive conditional heteroskedasticity. Journal of Econometrics, 74, 3-30.

Baillie, R. T., Han, Y. W. and Kwon, T-G. (2002). Further long memory properties of inflationary shocks. Southern Economic Journal, 3, 496-510.

Balicilar, M., Ozdemir, Z.A. and Cakan, E. (2011). On the nonlinear causality between inflation 
and inflation uncertainty in the G3 countries. Journal of Applied Economics, XIV (2), 269-296.

Balcilar, M. and Ozdemir, Z.A. (2013a). Asymmetric and time-varying causality between inflation and inflation uncertainty i n G -7 countries. Scottish Journal of Political Economy, 60 (1), 1-41.

Balcilar, M. and Ozdemir, Z. A. (2013b). The causal nexus between oil prices and equity market in the U.S.: A Regime Switching Model. Energy Economics, 39, 271-282.

Ball, L. (1992). Why does high inflation raise inflation uncertainty. Journal of Monetary Economics, 29, pp. 371-388.

Ball, L. and Mankiw, G.N. (1994). Asymmetric Price Adjustment and Economic Fluctuations. Economic Journal, 104, 247-261.

Barimah, A. and Amuakwa-Mensah, F. (2012) Does inflation uncertainty decrease with inflation? A GARCH model of inflation and inflation uncertainty for Ghana. West African Journal of Monetary \& Economic Integration, 12, 32-61.

Barimah, A. (2014) Exponential GARCH Modelling of the Inflation-Inflation Uncertainty Relationship for Ghana. Modern Economy, 5, 506-519Cukierman, A. and Meltzer, A. (1986). A theory of ambiguity, credibility, and inflation under discretion and asymmetric information. Econometrica, 54, 1099-1128.

Beran, J. (1995). Maximum likelihood estimation of the differencing parameter for invertible short and long memory autoregressive integrated moving average models. Journal of the Royal Statistical, Society, B 57,659-672.

Black, F. (1976). Studies in stock price volatility changes. Proceedings of the American Statistical Association, Business and Economics Statistics Section. American Statistical Association, 177-181.

Caporale, G. M., and Kontonikas, A. (2009). The Euro and inflation uncertainty in the European monetary union. Journal of International Money and Finance 28, 954-971.

Chen, S-W., Shen, C-H, and Xie, Z. (2008). Evidence of a nonlinear relationship between inflation and inflation uncertainty: The case of the four little dragons. Journal of Policy Modeling, 30, 363-376.

Chowdhury, A. (2014) Inflation and inflation-uncertainty in India: the policy implications of the relationship. Journal of Economic Studies, 41 Iss: 1, $71-86$.

Conrad, C. and Karanasos, M. (2005), On the inflation uncertainty hypothesis in the USA, Japan, and the UK: a dual long memory approach. Japan and the World Economy, 17, 327-343.

Cukierman, A. and Meltzer, A. (1986). A theory of ambiguity, credibility, and inflation under discretion and asymmetric information. Econometrica, 54, 1099-1128.

Davies, R. B. (1987). Hypothesis testing when a nuisance parameter is present only under the alternative. Biometrika, 74, 33-43.

Ding, Z., Granger, C. W. J. and Engle, R.F. (1993). A long memory property of stock market returns and a new model. Journal of Empirical Finance, 1, 83-106.

Eitrheim, $\emptyset$., and T.Teräsvirta (1996). Testing the adequacy of smooth transition autoregressive models. Journal of Econometrics, 74, 59-76.

Engle, R.F. and Ng, V.K. (1993). Measuring and Testing the Impact of News on Volatility. Journal of Finance, 5, 1749-1778.

Enders, W. and Hurn, S. (2002). Asymmetric price adjustment and the Phillips curve. Journal of Macroeconomics, 24, 395-412.

Fountas, S. and Karanasos, M. (2007). Inflation, output growth, and nominal and real uncertainty: Empirical evidence for the G7. Journal of International Money and Finance, 26, 229-50.

Franses, P.H., Bruin, P.D. and van Dijk, D. (2000). Seasonal Smooth Transition Autoregression. Econometric Institute Report 185. 
Friedman, M. (1977). Nobel lecture: inflation and unemployment. Journal of Political Economy, $85, \quad 451-472$.

Glosten, L.R., Jaganathan, R. and Runkle, D. (1993). On the Relation between the Expected Value and the Volatility of the Nominal Excess Return on Stocks. Journal of Finance, 48, 1779-1801.

Granger, C.W.J. (1993). Strategies for modelling nonlinear time-series relationships. The Economic Record 69, 233-238.

Granger, C.W.J. (1969). Investigating causal relations by econometric models and crossspectral methods. Econometrica, 37, 424-438.

Granger, C.W.J. and Joyeux, R. (1980). An introduction to long-memory time series models and fractional differencing. Journal of Time Series Analysis, 1, 15-39.

Granger, C.W.J., Engle, R.F. and Robins, R.P. (1986). Wholesale and retail prices: Bivariate time series modelling with forecastable error variances. In D. Belsley andE. Kuh, eds., Model Reliability, MIT Press, Cambridge, MA, 1-17.

Grier, K. B. and Perry, M. (2000). The effects of real and nominal uncertainty on inflation and output growth: some GARCH-M evidence. Journal of Applied Econometrics, 15, 45-58.

Grier, K. B. and Ye, H. (2007). Does high inflation predict greater inflation uncertainty? Evidence from asymmetric stochastic volatility models. manuscript, University of Oklahoma.

Hamilton, J. D. (1990). Analysis of Time Series Subject to Changes in Regime. Journal of Econometrics, 1-2, 39-70.

Hamilton, J. D. (1994). Time Series Analysis. Princeton, NJ: Princeton University Press.

Hasanov, M. and Omay, T. (2011). The relationship between inflation, output growth, and their uncertainties: Evidence from selected CEE countries. Emerging Markets Finance and Trade, Vol. 47 , Supplement 3, 5-20.

Hegerty, S. W. (2012). Does high inflation lead to increased inflation uncertainty? Evidence from nine African Countries. African Economic and Business Review 10(2), 1-15.

Holland, S. (1995). Inflation and uncertainty: tests for temporal ordering. Journal of Money, Credit, and Banking, 27, 827-837.

Hwang, Y. (2001). Relationship between inflation rate and inflation uncertainty. Economics Letters, 73, 179-86.

Jacobson, T., Lindh, T. and Warne, A. (2002). Growth, saving, financial markets, and Markov-Switching regimes. Studies in Nonlinear Dynamics and Econometrics, 5, 241-259.

Jiranyakul, K. and Opiela, T.P. (2010) Inflation and inflation uncertainty in the ASEAN-5 economies. Journal of Asian Economics 21, 105-112.

Karahan, O. (2012). The Relationship between Inflation and Inflation Uncertainty: Evidence from the Turkish Economy. International Conference of Applied Economics, Procedia Economics and Finance 1, $219-228$.

Karanasos, M., Karanassou, M. and Fountas, S. (2004). Analyzing US inflation by a GARCH model with simultaneous feedback. WSEAS Transactions on Information Science and Applications, 1, 767-72.

Kontonikas, A. (2004). Inflation and inflation uncertainty in the United Kingdom, evidence from GARCH modelling. Economic Modelling, 21, 525-543.

Krishnamurthy, V. and Rydén, T. (1998). Consistent estimation of linear and non-linear autoregressive models with Markov regime. Journal of Time Series Analysis, 19, 291-307.

Lindgren, G.(1978). Markov regime models for mixed distributions and switching regressions. Scandinavian Journal of Statistics, 5, 8191.

Luukkonen, R., Saikkonen, P., and Teräsvirta, T. (1988). Testing linearity against smooth transition autoregressive model. Biometrika, 75, 491-499. 
Mboweni, T. T. (2003). Inflation targeting in South Africa. BIS/South African Reserve Bank, management seminar dinner, Pretoria, 2nd September.

Nelson, D.B. (1991). Conditional Heteroscedasticity in Asset Returns: A New Approach. Econometrica, 59, 347-370.

Okun, A.M., (1971). The mirage of steady inflation. Brookings Papers on Economic Activity, 2, 485-498.

Oteng-Abayie, E.F. and Doe, S.K. (2013) Inflation and Inflation Uncertainty in Ghana. Journal of Business Management and Economics, 4, 259-266.

Ozdemir, Z. A. (2010). Dynamics of inflation, output growth and their uncertainty in the UK: an empirical analysis. The Manchester School, 78, 511-37.

Ozdemir, Z.A., and Fisunoğlu, M. (2008). On the Inflation-Uncertainty Hypothesis in Jordan, Philippines and Turkey: A Long Memory Approach. International Review of Economics \& Finance, 17, 1, 1-12.

Payne, J.E. (2008). Inflation and inflation uncertainty: evidence from the Caribbean region. Journal of Economic Studies, 35(6), 501-511.

Pourgerami, A. and Maskus, K. (1987). The effects of inflation on the predictability of price changes in Latin America: some estimates and policy implications. World Development, 15, 287-290.

Pretorius, K. (2012). The relationship between inflation, inflation uncertainty and economic growth in South Africa. MSc Thesis, University of Johannesburg, Johannesburg, South Africa.

Statistics South Africa (2014). P0141 - Consumer Price Index (CPI), May 2014. 18 June 2014.

Thornton, J. (2007). The relationship between inflation and inflation uncertainty in emerging market economies. Southern Economic Journal, 73(4), 858-870.

Trading Economics (2014). South Africa inflation rate. http://www.tradingeconomics.com/south-africa/inflation-cpi [Accessed 25 June 2014].

Ungar, M. and Zilberfarb, B. (1993). Inflation and its unpredictability-theory and empirical evidence. Journal of Money, Credit, and Banking, 25, 709-720.

Valdovinos, C.G.F. and Gerling. K. (2011). Inflation Uncertainty and Relative Price Variability in WAEMU Countries. IMF Working Paper, 59.

van der Merwe, E. J. (2004). Inflation targeting in South Africa. South African Reserve Bank, Occasional paper No. 19.

van Dijk, D., Franses, P.H., and R. Paap (2002). A nonlinear long memory model for US unemployment. Journal of Econometrics, 102, 135-165.

Viorica, D., Jemna, D., Pintilescu, C. and Asandului, M. (2014). The relationship between inflation and inflation uncertainty: Empirical evidence for the newest EU countries. PLoS ONE, 9(3), $1-8$.

Warne, A. (2000). Causality and Regime Inference in a Markov Switching VAR. Working Paper Series, 118, Sveriges Riksbank (Central Bank of Sweden). 